AIAA 2002-2604

\title{
AEROACOUSTIC MEASUREMENTS OF A WING/SLAT MODEL
}

\author{
Jeff M. Mendoza * \\ Honeywell Engines, Systems, and Services \\ Phoenix, AZ \\ Thomas F. Brooks ${ }^{\dagger}$ \\ William M. Humphreys $\ddagger$ \\ NASA Langley Research Center \\ Hampton, VA
}

\begin{abstract}
Aeroacoustic evaluations of high-lift devices have been carried out in the Quiet Flow Facility of the NASA Langley Research Center. The present paper deals with detailed flow and acoustic measurements that have been made to understand, and to possibly predict and reduce, the noise from a wing leading edge slat configuration. The acoustic database is obtained by a moveable Small Aperture Directional Array (SADA) of microphones designed to electronically steer to different portions of models under study. The slat is shown to be a uniform distributed noise source. The data was processed such that spectra and directivity were determined with respect to a one-foot span of slat. The spectra are normalized in various fashions to demonstrate slat noise character. In order to equate portions of the spectra to different slat noise components, trailing edge noise predictions using measured slat boundary layer parameters as inputs are compared to the measured slat noise spectra.

\section{List of Symbols}

$\mathrm{C}_{\mathrm{p}} \quad$ Coefficient of pressure

$\hat{e} \quad$ Column steering vectors

QFF Quiet Flow Facility

$\mathrm{F} \quad$ Transfer function for per-foot processing

$\hat{G} \quad$ Cross-spectral matrix

h Trailing edge thickness

* Senior Engineer, Acoustics, Member AIAA

+ Senior Research Scientist, Aeroacoustics Branch, Associate Fellow AIAA

$\ddagger$ Research Scientist, Advanced Measurement and Diagnostics branch, Senior Member AlAA

Copyright $\mathcal{O} 2002$ by the American Institute of Aeronautics and Astronautics, Inc. No copyright is asserted in the United States under Title 17, U.S. Code. The U.S. Government has a royalty-free license to exercise all rights under the copyright claimed herein for Government Purposes. All other rights are reserved by the copyright owner.
\end{abstract}

\begin{tabular}{|c|c|}
\hline $\mathrm{m}$ & Microphone number \\
\hline M & Freestream flow Mach number \\
\hline 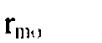 & Microphone to source distance \\
\hline SADA & Small Aperture Directional Array \\
\hline TE & Trailing edge \\
\hline $\mathrm{BL}$ & Boundary layer \\
\hline u & Local flow velocity \\
\hline $\mathbf{u}_{\mathrm{sv}}$ & Average slat trailing edge velocity \\
\hline$u_{\mathrm{g}}$ & Gap velocity \\
\hline$u_{p}$ & Pressure side slat TE velocity at $\delta_{p}$ \\
\hline$u_{s}$ & Suction side slat TE velocity at $\delta_{\mathrm{s}}$ \\
\hline $\mathrm{U}_{\infty}$ & Free stream (open-jet) velocity \\
\hline$w_{\mathrm{in}}$ & Frequency dependent weighting function \\
\hline$W$ & Row matrix of $w_{\mathrm{m}}$ terms \\
\hline$w_{\mathrm{m}}$ & SADA weighting factors \\
\hline$\alpha_{111}$ & Main element angle of attack \\
\hline$\alpha$ & $\begin{array}{l}\text { Slat angle of attack relative to main-element } \\
\text { waterline }\end{array}$ \\
\hline$\delta_{\mathrm{p}}$ & Pressure side BL thickness \\
\hline$\delta_{p}^{*}$ & Pressure side BL displacement thickness \\
\hline$\delta_{s}$ & Suction side BL thickness \\
\hline$\delta_{s}^{*}$ & Suction side BL displacement thickness \\
\hline$\Psi$ & Array azimuth (sideline) angle \\
\hline$\Phi$ & Array elevation (over-flight) angle \\
\hline$\Phi_{\text {tatj }}$ & $\begin{array}{l}\text { Elevation angle adjusted for shear layer } \\
\text { effects, emission angle in retarded coordinates }\end{array}$ \\
\hline & Introduction \\
\hline
\end{tabular}

Background

With the advent of high by-pass ratio fans and the rapid advances of low noise engine technologies, airframe noise sources can now dominate the aircraft noise signature under high lift conditions(Macaraeg'). On approach, airframe noise can be a primary offender. The airframe landing gear, flaps and flap side edges, and the leading-edge slat have been identified as key airframe noise sources (Davy and Remy ${ }^{2}$, Hayes et al. ${ }^{3}$, Dobrzynski et al. ${ }^{4}$, Guo et al. ${ }^{56}$, etc.). The relative importance and dominance of these noise sources over different frequency ranges depend on the trimmed airframe configuration. Progress has been made in 
understanding, prediction capability, in reducing airframe noise under, first, the NASA Advanced Subsonic Technology (AST) Program and, now, the NASA Quiet Aircraft Technology (QAT) program. The present paper reports results of slat noise measurements conducted in the Quiet Flow Facility (QFF) at NASA Langley Research Center.

The leading edge slat of a multi-element wing is designed to delay the onset of main element separation by alleviating the suction side pressure peak of the main element leading edge (see Figure 1 for key terminology). This is achieved by accelerating flow through the slat gap that results in an increase of the wing $C_{\text {Imix }}$. More detailed descriptions of the complex flow field between the slat and main element would involve the viscous interactions of the slat wake and the main element boundary layer. With regard to operating configurations, one may anticipate that trade-offs would occur between control of acoustic emissions and that of aerodynamic performance. For example, although it has been suggested ${ }^{7}$ that reducing the slat gap (demonstrated in the present measurements also) can attain slat noise reduction, reduced lift performance can be a consequence ${ }^{8}$. The gap flow field is believed to be central to the slat noise (and performance) problem. Moriarty et. al. ${ }^{9}$ measured key flow characteristics from a slat and main element model using PIV measurements. Velocities through the slat gap were found to be as high as twice the freestream value. In addition, turbulent kinetic energy levels were highest in the slat gap region where the separating shear layer reattaches to the backside of the slat. This suggests that this region is likely to be the most energetic if feedback from the slat trailing edge amplifies disturbances.

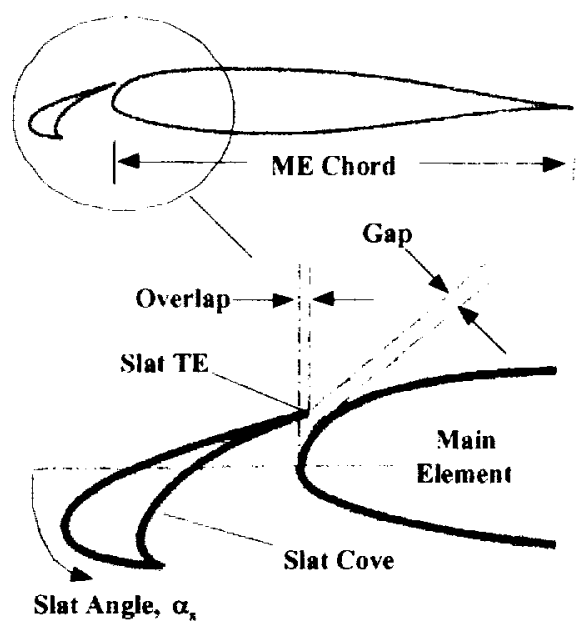

Figure 1 Schematic of a leading edge slat and wing main element leading edge.
Some early airframe noise investigations sought to separate airframe noise contributions from a $4.7 \%$ scale DC-10 model. With the reduction of localized flap sources using control devices, they were able to observe the slat noise contribution to the overall noise. Their phased array results also identified the distributed nature of the leading edge slat source. Guo et al. ${ }^{56}$ also utilized the same model to define flat conditions which appear to result in significant slat noise. The flap and slat are independent noise sources that would only affect one another through their mean aerodynamic influence.

In addition to full aircraft modeling, specific airframe component noise has been the subject of a number airframe noise studies. Dobrzynski et al. ${ }^{4}$ used a $1 / 10^{\text {th }}$ scaled Airbus-type high lift wing to quantify wing noise sources. They found that under certain slat configurations vortex shedding from the slat edge cove resulted in excessive tonal noise in the vicinity of the slat. These tones were the result of laminar shedding off the slat cove and were reduced by boundary layer tripping just upstream of the cove leading edge. Trailing edge (TE) noise was also identified as a significant slat source and scaled to the 5 th power of the freestream velocity. The wing model used in this investigation included a slat track where noise levels in the vicinity of the slat tracks or supports were typically $\sim 8-10 \mathrm{~dB}$ higher locally than the levels in other slat regions. The effect of which could not be entirely dismissed from the total noise spectra for the slat region. Similarly, Storms, et al. ${ }^{10}$ focused their aeroacoustic measurements on the leading edge slat using a high lift model consisting of several slat brackets, a main element, and a partial span outboard flap. Their large array and their processing produced phased array noise maps containing highly localized noise sources across the span of the slat and therefore could not accurately focus on other key slat noise mechanisms. Their RANS computations and measurements indicated the potential for KelvinHelmholtz instabilities in the slat cove that grows as the flow is accelerated through the slat gap. It was conjectured that these instabilities could be part of a feedback mechanism that included the slat trailing edge vortex shedding.

A number of computational models have been developed to describe the slat noise generation and radiation problem (Guo ${ }^{7,11}$, Khorrami, et. al. ${ }^{12}$, Singer et. al. ${ }^{13}$, and Khorrami et. al. ${ }^{14}$ ). Most utilizing RANS techniques to capture specific flow features pertaining to the slat cove and trailing edge. Qualitative agreement with measured data was reasonably found ${ }^{12,13.14}$. Guo's ${ }^{7}$ recent slat noise modeling publication utilizes a discrete vortex modeling approach 
to capture the unsteady flow field around the cusp and through the slat gap and subsequent acoustic interactions. The most notable results of this work are the documented slat noise radiation and Mach number scaling relation. Guo reported that for low frequency slat noise the dominant radiation direction is normal to the slat element chord and that slat trailing edge sources should scale as $\mathrm{M}^{5}$. Cove region vortical fluctuations resulted in energy radiating dominantly in the fly-over direction and normal to the main element chord. Analysis was presented indicating this mechanism could scale as $\mathrm{M}^{2.5}$ as justified by computation for noncompact sources at high frequencies.

The recent work of Dobrzynski et. al. ${ }^{15}$ is a comprehensive presentation of slat noise measurements including scaling results and directivity. Their measured noise source amplitudes scaled as the 4.5 power of the wind tunnel velocity and the frequency scaled with the slat cove vortex dimension (approximated using the slat chord length) and wind tunnel velocity. Their directivity results support a complex dipole model of slat trailing edge noise with maximum radiation in the rear arc direction.

\section{Present Approach}

This paper discusses the results of 2-dimensional wing/slat model tests conducted in the Quiet Flow Facility (QFF). Data obtained from a Small Aperture Directional Array (SADA) of microphones positioned about the model, static pressure measurements on the model, boundary layer probe measurements in the wake of the slat, and unsteady pressure sensors on the slat and main element surfaces are used to characterize slat noise. To better isolate the true distributed slat noise source from extraneous sideplate juncture noise sources, additional processing steps are added to our standard beamforming approach. The resultant noise spectra are representative of noise from a slat with a one-foot span. This allows an examination of scaling laws, directivity, and diagnostic prediction comparisons.

\section{Airframe Component Test and Processing}

\section{Test Set-Up and Method}

The testing of various wing / slat geometries was conducted in the NASA Langley Quiet Flow Facility (QFF). The setup is shown in Figure 2. The QFF is an open-jet facility designed for anechoic acoustic testing. For this study, a 2- by 3-foot rectangular open-jet nozzle was employed which provided up to a 0.17 Mach number flow. The 3-foot span model had a 16" chord $\left(6 \%\right.$ of full-scale) NACA $63_{2}-215$ main element airfoil with a slat chord of 3.2 ".

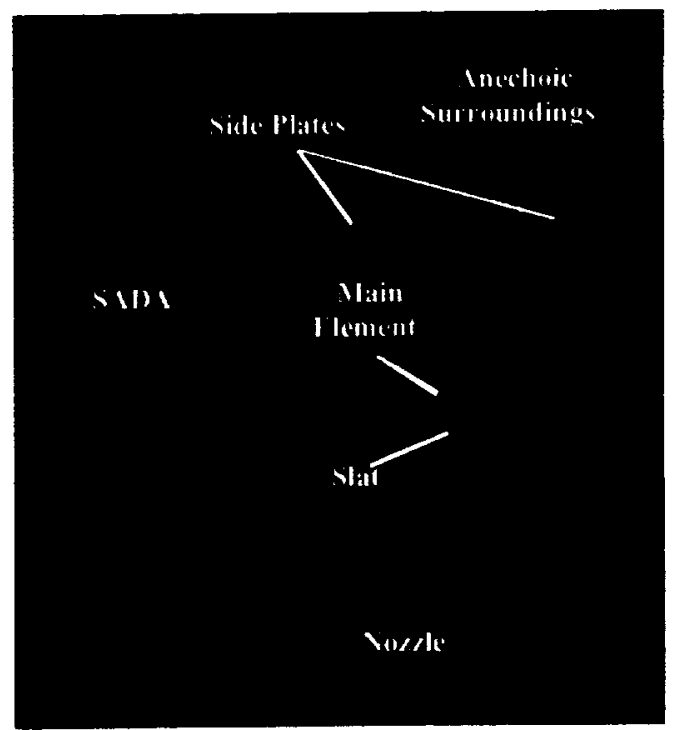

Figure 2 Wing/slat test apparatus in QFF.

The main element and slat were instrumented with static pressure ports and unsteady pressure transducers. The model was held in place via the use of vertical side plates, which were aligned and rigidly fastened to the exit of the nozzle. Appropriate acoustic foam treatments were applied to all edges and supports to reduce acoustic reflections from these surfaces. The slat geometric positions referenced to the main element are shown in Figure 3. The slat at each angle of $\alpha_{s}=$ $10^{\circ}, 20^{\circ}$, and $30^{\circ}$ is pivoted about a single trailing edge "notch" position of zero for the baseline case. The other notch positions, which are closer to the main element and thus serve to close off the gap, are shown. Table 1 gives the slat overlap and gap dependence on notch position. For the data presented, the main element was aligned at geometric angles of attack of $26^{\circ}$ and $32^{\circ}$ relative to the undisturbed flow. These large physical angles were used, in this open-jet test setup, to duplicate the slat-area aerodynamics typical of high-lift wing / slat configurations. This method is fully discussed in the subsequent model aerodynamics section. 


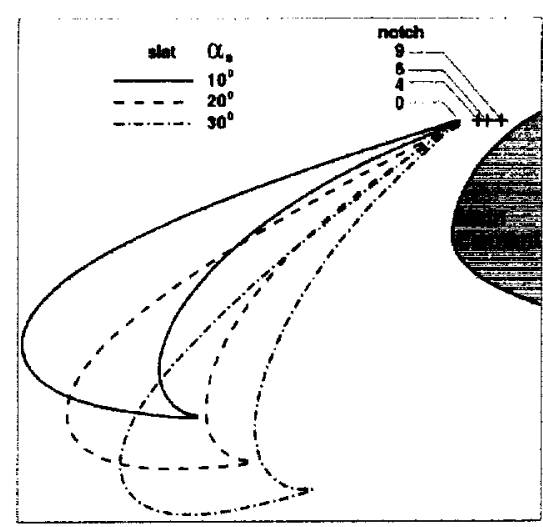

Figure 3 Slat deflections and notch settings.

\begin{tabular}{|c|c|c|}
\hline $\begin{array}{c}\text { Slat Position } \\
\text { (notches) }\end{array}$ & Overlap/C \% & Gap/C \% \\
\hline 0 & 0.307 & 1.713 \\
\hline 4 & 1.188 & 1.084 \\
\hline 6 & 1.563 & 0.833 \\
\hline 8 & 1.941 & 0.591 \\
\hline 9 & 2.126 & 0.476 \\
\hline
\end{tabular}

Table 1 Overlap and Gap percentages of chord for $20^{\circ}$ slat condition. For the $10^{\circ}$ slat, add $0.016 \%$ to all Overlap numbers and add $0.09 \%$ to all Gap numbers. For the $30^{\circ}$ slat, subtract $0.061 \%$ and add $0.140 \%$, respectively.

Acoustic directivity and spectra of selected portions of the wing-slat model were obtained using the Langley-developed SADA. The SADA consisted of 33 B\&K model 4138, 1/8-inch microphones with 1/4-inch preamplifiers projecting from an acoustically treated aluminum frame. The array pattern incorporated four irregular circles of eight microphones each with one microphone placed at the center of the array. Each circle was twice the diameter of the circle it enclosed. The maximum radius of the array was 3.89 inches. Two small laser diode pointers were incorporated into the array frame on opposite sides of the center microphone for use in alignment.

The SADA was mounted on a pivotal boom designed such that the array could be positioned at a number of elevation and azimuth angles. While the boom pivot was not centered with the slat, the electronic steering processing fully accounted for array orientations and positions. Rotation of the boom was performed using precision DC servo rotation stages. Additional details concerning array and boom construction can be found in References 16 and 17 .

Dynamic surface pressures were obtained along the centerline chord of the slat and the leading edge of the main element airfoil using Kulite model LQ-34-064-5A sensors. The positions of the flush-mounted sensors are shown in Figure 4. The chordwise distance from the leading edge is $x$ and the spanwise distance from the center of the span is $y$. The chordwise position for each slat sensor at a main element angle of attack of $26^{\circ}$ and a slat angle of $10^{\circ}$ is given in Table 2 .

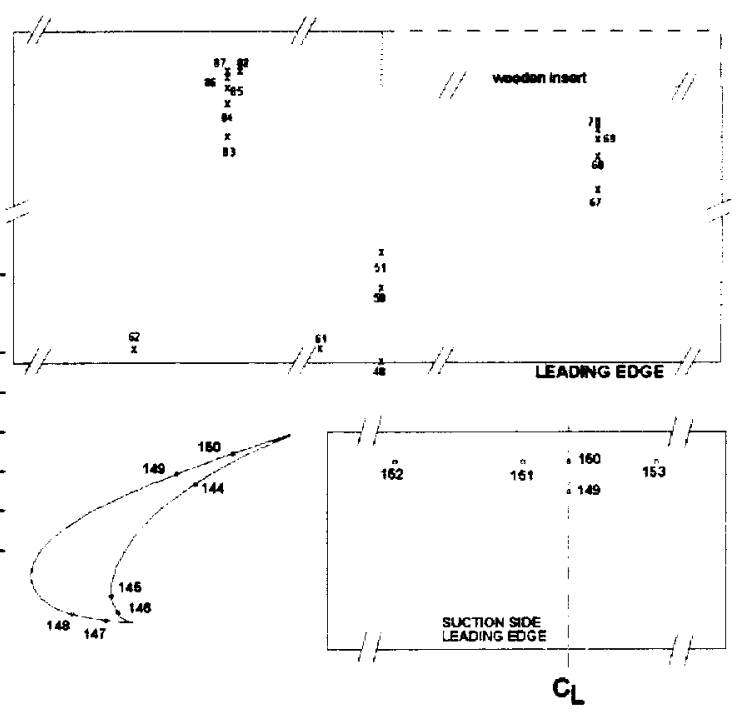

Figure 4 Unsteady pressure sensor distribution.

\begin{tabular}{l|lll} 
Kulite \# & $\mathbf{X}$ & $\mathbf{Y}$ & $\mathbf{Z}$ \\
\hline 144 & 23.51 & 0.00 & 0.16 \\
145 & 22.12 & 0.00 & -0.50 \\
146 & 22.11 & 0.00 & -0.70 \\
147 & 21.95 & 0.00 & -0.71 \\
148 & 21.63 & 0.00 & -0.48 \\
149 & 23.36 & 0.00 & 0.35 \\
150 & 24.04 & 0.00 & 0.26 \\
151 & 24.04 & 0.53 & 0.26 \\
152 & 24.04 & 2.00 & 0.26 \\
153 & 24.04 & -1.00 & 0.26
\end{tabular}

Table 2 Sensor coordinates in tunnel coordinates (inchs).

\section{Data Acquisition and Post Processing}

The acquisition hardware used to collect data from the array microphones and surface pressure sensors consisted of a series of transient data recorders controlled by a workstation. Signals were simultaneously recorded at a sampling rate of 142.857 $\mathrm{kHz}$, with 14 bits dynamic range for the 33 SADA microphones and with 12 bits for the surface pressure sensors, Two million 2-byte samples were collected for each acquisition. The microphone signals were high pass filtered at $300 \mathrm{~Hz}$, and all channels employed antialiasing filters set to $50 \mathrm{kHz}$. 
Microphone and surface pressure sensor calibration data were accounted for in the post-processing. For the SADA microphones, pistonphone and injection calibrations of amplitude and phase were conducted. Amplitude and phase calibrations for the surface pressure sensors were obtained using a miniature acoustic source capable of high frequency output that was referenced to the output of a B\&K 4138 microphone.

Data processing of SADA microphone signals included the construction of cross-spectral matrices from the raw time data. The individual elements in each cross-spectral matrix were computed using Fast Fourier Transforms (FFT's) of the original data ensemble using a Hamming window. These data were segmented into 1000 non-overlapping blocks each containing $2^{13}$ samples yielding a frequency resolution of $17.44 \mathrm{~Hz}$. Classical beamforming was performed on the crossspectral matrices to electronically "steer" the SADA to predefined noise source locations using the equation

$$
P(\hat{e})=\frac{\hat{e} T \hat{W} \hat{G} \hat{W}^{T} \hat{e}}{\left(\sum_{m=1}^{m_{m}} w_{m}\right)^{2}}
$$

where $w_{m}$ is a frequency dependent weighting function obtained via a unique shading algorithm providing a constant beamwidth independent of frequency (between 10 to $40 \mathrm{KHz}$ ). $\hat{W}$ is a row matrix containing the $w_{m}$ terms, $\hat{G}$ is a cross spectral matrix, and $\hat{e}$ is a column steering vector containing terms for each microphone in the array. The output of equation (1) is the meansquared-pressure $P$ measured at the steering location. Mean amplitude and phase changes due to refracted sound transmission through the curved shear layer associated with the mounting of the model in the QFF were corrected and incorporated into the steering vector using Snell's law in Amiet's method. A typical mean refracted ray path is shown in Figure 5. Using equation (1), noise source distribution maps, spectra, and directivity were generated for a number of test conditions. Beamforming results were obtained at a number of pressure side elevation angle positions, which are shown on the right-hand side in Figure 6. Additional details concerning the SADA, beamforming method, shear correction, and shading algorithm can be found in References 16 and 17.

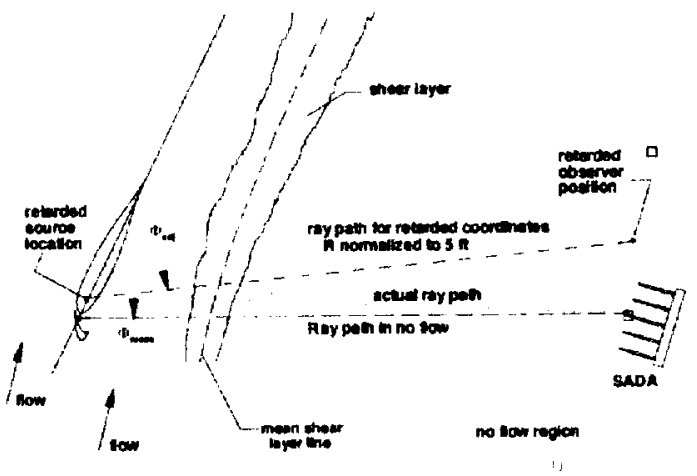

Figure 5 Typical mean reflected ray path.

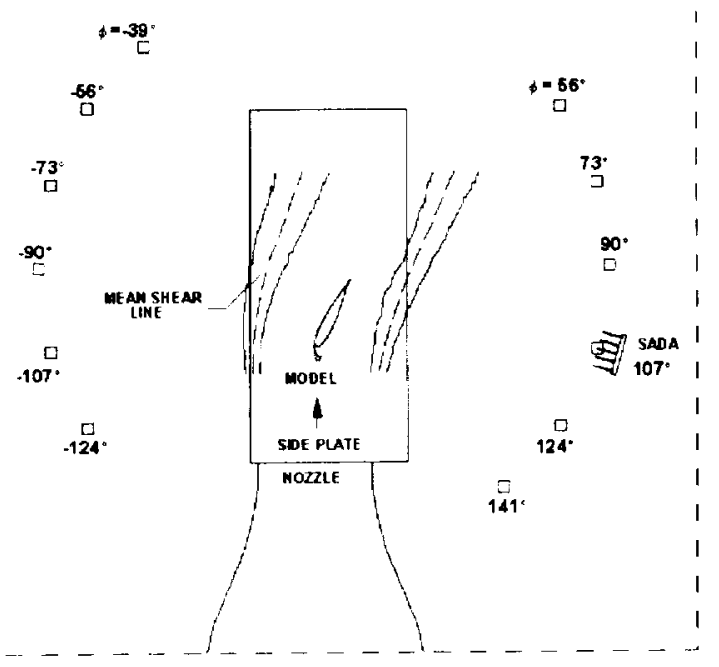

Figure 6 SADA measurement elevation angles.

\section{Array Processing of Distributed Source Noise}

A spanwise uniform flowfield over a wing and slat consisting of a pressure side slat cove and a gap between the main element and slat trailing edge will inherently produce a uniform line distribution of independent sources of noise (independent to the extent that the correlated turbulence scales are small compared to the span). In reality, the slat and main element wing are coupled by bracket structures that in some aircraft configurations can be complex and bulky, adding to the complexity of the noise sources. In the present QFF model configuration, the slat and the main element are both mounted to sideplates. Because both the slat and main element are lifting surfaces and the sideplates are not, localized vortical flow occurs near the junctures, whose interaction with the surfaces produce localized noise sources. It is the goal of the present study to examine only the uniform slat noise. For this paper a processing method is used that extracts the slat noise while suppressing the juncture noise contributions. 
Figure 7 shows a typical noise map from SADA to illustrate the presence of both the distributed source and localized juncture noise sources at a particular frequency $(12.5 \mathrm{kHz})$. For clarity, only the highest 10 $\mathrm{dB}$ contour levels are shown. A contour value at a point is the SADA output when steered to the point along a plane defined through the main element chord line. The distributed slat noise source is clearly seen along the center portion of the span. It is desired to determine spectra representing the slat noise produced by one foot of span. One possible processing method would be to incorporate the contour integration method of Reference 18. However, since a requirement of this integration method is that the source region of all major contributors to any contour area under consideration be included in the integration, one would have to determine the total noise from both the distributed slat noise and the juncture noise sources. From this total noise, one would have to subtract the juncture noise contributions. This would involve source-subtraction modeling that is not yet accomplished. Instead, a simpler method is used that takes advantage of a design (and standard processing) feature of the SADA that the main-sensing-lobe "3 dB down" spatial resolution is approximately one foot in width and that any noise contributions from the side-plate juncture are minimized when the SADA steered to the span center. This is true for a set frequency range of $10-40 \mathrm{kHz}$. At frequencies below $10 \mathrm{kHz}$, the main lobe widens. Above $40 \mathrm{kHz}$, the main lobe narrows. The extraction method used here employs this standard SADA processing and then adds additional steps to more exactly account for array characteristics in the final determination of a per-foot noise spectrum.

The processing procedure used for this QFF configuration involves first beamforming the SADA to the center of the slat noise region (this location is verified by examining maximum contour levels in the streamwise direction along the span centerline in the slat region for $12.5 \mathrm{kHz}$ ). A full spectrum is generated using standard SADA processing. The spectrum is then adjusted in amplitude as a function of frequency by the function $F$. This function is the ratio of the noise that would be perceived by a single microphone at the SADA location from a uniform distribution of independent noise sources distributed over a 1 -foot span and the noise that would be perceived by the SADA from a similar uniform source but distributed over a 3.25-foot span. Thus the adjusted measured spectrum would represent the "per-foot" spectrum of the slat noise alone. The function $F$ is determined based on SADA response as calculated for each SADA position and orientation with respect to the slat. The extra 0.25 -foot in span is added to approximately account for reflection effects from the sideplates. In comparing the basic SADA spectra to the adjusted spectra, one finds as expected that the levels between frequency range of $10-40 \mathrm{kHz}$ nearly match and that the levels of the adjusted spectra are lower at frequencies less than $10 \mathrm{kHz}$ because of the array main lobe broadening of which the $F$ function accounts for. A possible source of error in this procedure is that sideplate juncture noise, of which the SADA suppresses between 10 to $40 \mathrm{kHz}$, is less suppressed at lower frequencies, however such positive level bias is believed to be small as contour plots for lower frequencies reveal less obvious sideplate contribution.

The narrowband beamformed spectra were determined over a frequency range of $300-50000 \mathrm{~Hz}$ using a frequency step size of $17.45 \mathrm{~Hz}$. After applying the $F$ function, one-third Octave spectra were formed and were then normalized to a five-foot observer distance. The processing produced spectra representing slat noise from a 1-foot spanwise section, as measured by a signal microphone 5 feet from the center of the span.

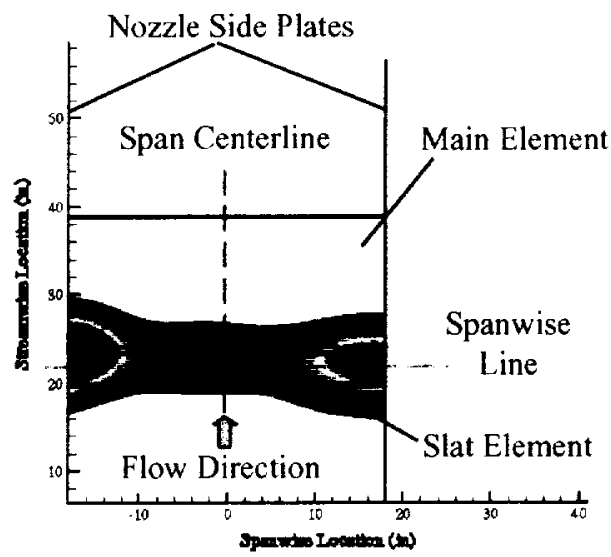

Figure 7 Pressure side (flyover) view of distributed slat noise with local discontinuities at the side plates.

\section{Model Aerodynamics}

Because of the relative size of the open jet wind tunnel test section compared to that of the model, one could not properly test the complete slat / main element /flap high lift configuration. Ideally from an aerodynamic simulation standpoint, the testing for a complete model of this size would be conducted in a larger tunnel, but because of the significant acoustic measurement advantages for this QFF test arrangement, a practical testing approach was taken. Following the testing philosophy used previously in studies ${ }^{17,18,19}$ of the flap for this model, the model was tested such as the local aerodynamics could be properly matched to represent high-lift device conditions. This is permitted acoustically because of the independence of noise 
source regions. In fact, one benefit of this approach is that, because the slat, main element, and flap were not tested simultaneously, less noise sources are present and thus the interpretation of the acoustic measurements is simplified.

The model was instrumented with static pressure tabs on both the slat and main element to define the model aerodynamics in the slat region. The flap was removed from the model. Large main element angles of attack were used to compensate for the lack of lift typically generated by a main element and flap. The approach attained high-lift aerodynamics about the slat region, while knowingly compromising the main element aerodynamics downstream of approximately the quarter chord.

The pressure $C_{p}$ distributions about the slat and leading edge region of the main element is shown in Figure 8 for the two main element angles and the three slat angles tested. The slat notch position was zero (see Figure 3 and Table 1) and the tunnel Mach number was 0.17 . As seen, there are a relatively small number of sensors on both the slat and the main element particularly in the trailing edge of the slat and the leading edge of the main element regions. Still the anticipated high lift device character is clearly shown. For increasing slat deflection angle, the trends are for decreasing slat leading-edge suction peak and increasing slat trailing-edge suction (indicative of increasing gap velocity). If one compared the present $C_{p}$ results to that of Storms, et $a^{10}$, our slat region aerodynamics for $\alpha_{m}=26^{\circ}$ and $32^{\circ}$, correspond to approximately their $\alpha_{\mathrm{m}}=4^{\circ}$ and to one less than their $8^{\circ}$, respectively. Storms, et al. tested beyond $\alpha_{\mathrm{m}}=16^{\circ}$, so our loading was on the lighter end of their testing range.

Accurate estimates of velocities in and about the slat trailing edge were not possible using the $C_{p}$ data because of the very sparse distribution of pressure ports near the edge and on the main element. However, even if one had a fine distribution, one would not be able to determine boundary layer / near wake characteristics important for evaluating the slat noise problem. Therefore, velocity survey measurements were made across the near wake of the slat trailing edge. The measurements were made using a specially constructed Pitot tube probe that consisted of parallel tube components. The probe orientations and respective traverse paths that were used are illustrated in an inset of Figure 9. The traverse path being aligned perpendicular to the main element surface is the probe orientation used for most data presented in this paper. The probe's outer diameter was .030", the inside diameter was .018 ". The survey path brought the probe to within .010". This closeness was obtained by final positioning of the probe path after the probe deflection was established within the flow.

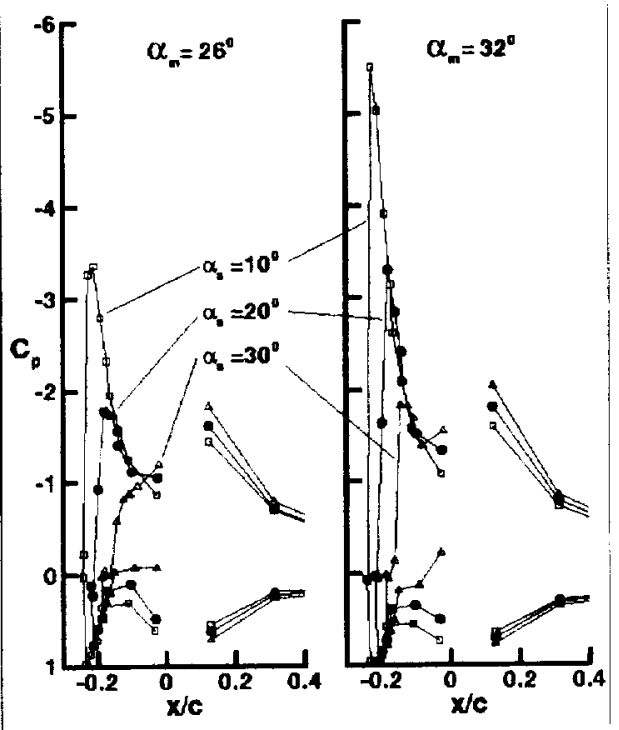

Figure 8 Slat and main element $C_{p}$ distributions.

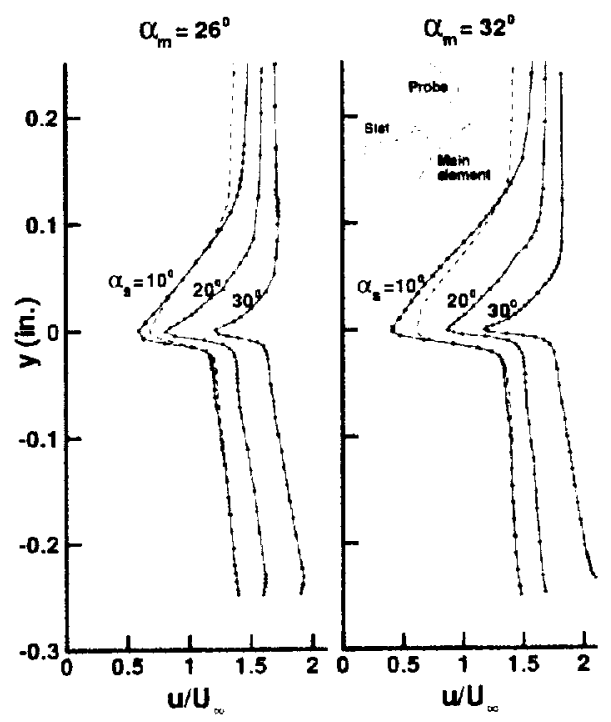

Figure 9 Slat trailing edge wake surveys.

The velocity survey results for the zero-notch reference configurations are shown in Figure 9. The y' axis is along the traverse path with positive $y$ ' on the slat suction side and negative $y^{\prime}$ on the pressure side / gap region. The data shown for slat angles $\alpha_{s}=10^{\circ}$, $20^{\circ}$, and $30^{\circ}$ are for the probe path aligned perpendicular to the main element surface. The $y^{\prime}=0$ position was defined as corresponding to the minimum velocity value. To show the effect of probe orientation, data for $\alpha_{\mathrm{s}}=10^{\circ}$ are shown for the probe path aligned 
perpendicular to the slat surface trailing-edge mean line. The actual velocity vector orientations, which affect these velocity amplitude measurements over the survey distance, were not determined. In general, it is seen that the velocities are higher for the larger main element angles and higher for the larger slat angles. The larger slat angles have smaller boundary layers on both the suction and pressure sides.

The slat trailing edge boundary layer / near-wake thicknesses $\delta_{\mathrm{s}}$ and $\delta_{p}$ of the suction and pressure sides, respectively, were chosen based (somewhat subjectively) on the curvature of the velocity survey results. These values of $\delta_{\mathrm{s}}$ and $\delta_{\mathrm{p}}$, along with their corresponding velocities $u_{\mathrm{s}}$ and $u_{\mathrm{p}}$, are show in Figure 10 to show the dependence of slat trailing edge flow on angles $\alpha_{n 1}$ and $\alpha_{s}$, and gap opening. The square symbols, representing the values determined from Figure 9, show readily the trends already sited of increasing velocity and decreasing boundary layer values for larger slat angles. For each slat and main element angle condition, decreasing the gap (increasing the notch number, see Figure 3 ) is seen here to change the velocities somewhat. Also shown are the $\delta_{\mathrm{s}}$ and $\delta_{\mathrm{p}}$ values determined using the alternate velocity survey path data of Figure 9 for $\alpha_{s}=10^{\circ}$. Some differences can be seen as a result of the different orientation.

Subsequently, use is made of the values of displacement thicknesses $\delta_{s}^{*}$ and $\delta_{p}{ }^{*}$. For the baseline cases, these are given in Table 3 along with $\delta_{\mathrm{s}}$ and $\delta_{\mathrm{p}}$, and the corresponding velocities $u_{s}$ and $u_{p}$. The $\delta^{*}$ 's were determined by integration from $y^{\prime}=0$ to the respective $\delta$ values. Any error in the $\delta^{*}$ values due to lack of resolution because of probe size and distance from the edge, should be offset due to ignoring the trailing edge thickness in the $\delta^{*}$ calculations.

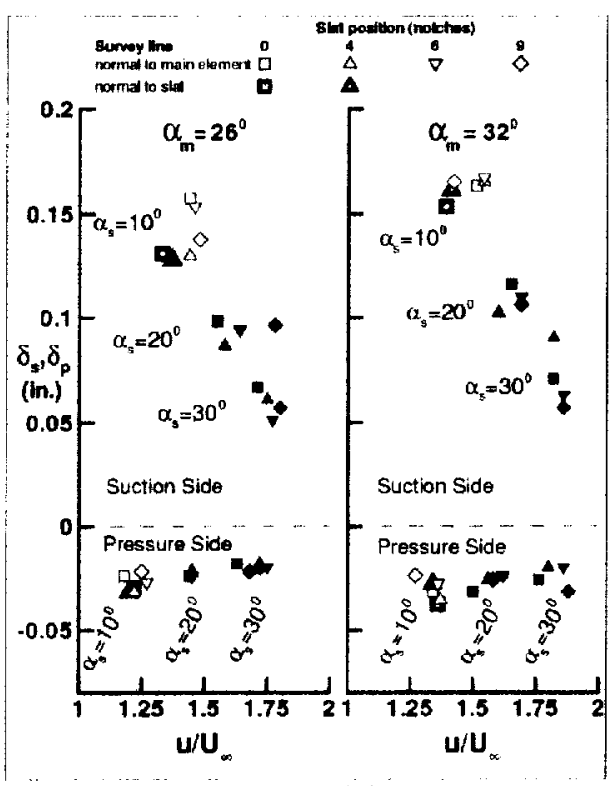

Figure 10 Boundary layer thicknesses summary as a function of relative local velocity.

\begin{tabular}{|c|c|c|c|c|c|c|c|c|c|}
\hline $\boldsymbol{\alpha}_{\mathbf{m}}{ }^{\circ}$ & $\boldsymbol{\alpha}_{\mathbf{s}}{ }^{*}$ & $\boldsymbol{\delta}_{\mathbf{S}}$ (in.) & $\boldsymbol{\delta}_{\mathbf{s}}$ (in.) & $\boldsymbol{\delta}_{\mathbf{P}}$ (in.) & $\boldsymbol{\delta}^{*}{ }_{\mathbf{P}}$ (in.) & $\mathbf{u}_{\mathbf{S}} / \mathbf{U}_{\infty}$ & $\mathbf{u}_{\mathbf{P}} / \mathbf{U}_{\infty}$ & $\mathbf{u}_{\mathbf{a v}} / \mathbf{U}_{\infty}$ & $\mathbf{u}_{\mathrm{g}} / \mathbf{U}_{\infty}$ \\
\hline 26 & 10 & .158 & .0355 & .024 & .0065 & 1.44 & 1.18 & 1.31 & 1.28 \\
\hline 26 & 20 & .098 & .0144 & .024 & .0032 & 1.55 & 1.44 & 1.50 & 1.55 \\
\hline 26 & 30 & .067 & .0056 & .018 & .0021 & 1.71 & 1.63 & 1.67 & 1.75 \\
\hline 32 & 10 & .159 & .0563 & .032 & .0080 & 1.51 & 1.34 & 1.43 & 1.40 \\
\hline 32 & 20 & .116 & .0212 & .032 & .0031 & 1.65 & 1.50 & 1.58 & 1.60 \\
\hline 32 & 30 & .071 & .0085 & .026 & .0022 & 1.82 & 1.76 & 1.79 & 1.90 \\
\hline
\end{tabular}

Table 3 Determined values of TE near-wake thicknesses, displacement thicknesses, and associated velocities for slat at notch position of zero. $\mathrm{M}=\mathrm{U}_{\infty} / \mathrm{c}=\mathbf{0 . 1 7}$.

\section{Results and Discussion}

\section{Slat and Main Element Angle Variations}

Figure 11 illustrates the effect of slat angle of attack on far field noise for $\alpha_{m 1}=26^{\circ}$ and $M=0.17$. The low frequency noise levels $(<10 \mathrm{kHz}$ ) remain relatively unchanged as the slat angle is increased from $10^{\circ}$ to $20^{\circ}$ but somewhat more at the $30^{\circ}$ slat deflection. Above
$10 \mathrm{kHz}$ the presence of a broadband hump develops with the peak increasing in level and slightly in frequency as the slat deflection angle is increased. This same basic noise trend is observed for the varying slat deflection angles with the main element setting now at $32^{\circ}$, see Figure 12. Again, low frequencies are relatively unchanged while the high frequency 
broadband spectral hump is present, but only for $\alpha_{s}=$ $30^{\circ}$.

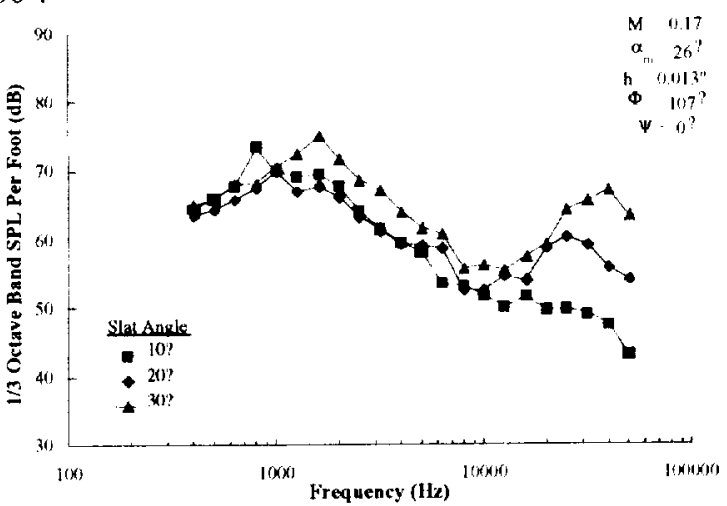

Figure 11 Effect of slat deflection for $\alpha_{m}=26^{\circ}$.

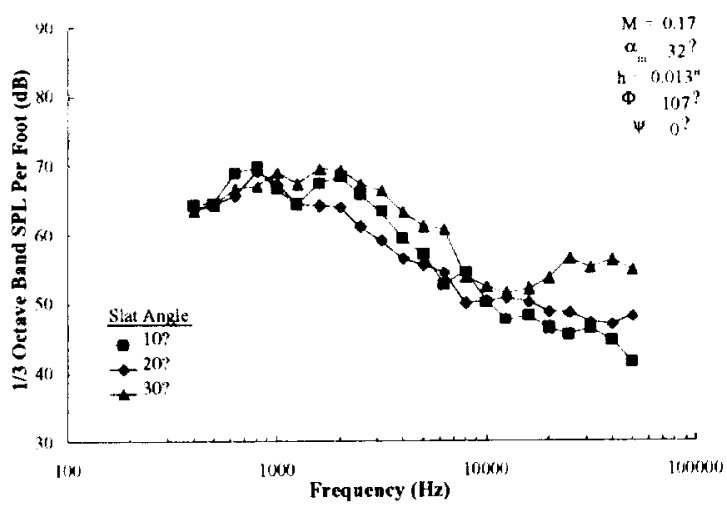

Figure 12 Effect of slat deflection for $\alpha_{n}=32^{\circ}$.

As will be illustrated in the section discussing trailing edge bluntness effects, this high frequency noise increase is not likely the result of trailing edge bluntness noise. It does however appear that it is related to changes in local flow velocities. In a later section it is demonstrated that the high frequency noise may be due to turbulent boundary layer trailing edge (TBL-TE) scattering from the pressure side of the slat. As seen from Figure 11, there is an increase in the high frequency broadband hump of $\sim 10 \mathrm{~dB}$ and $\sim 6 \mathrm{~dB}$ going from $10^{\circ}$ to $20^{\circ}$ and $20^{\circ}$ to $30^{\circ}$, respectively. The increase in gap velocity follows a similar trend as the noise where, from Table 3 , the larger increase in gap velocity occurs between the $10^{\circ}$ and $20^{\circ}$ slat deflection cases. The higher gap velocities result in thinner bounder layers that are known to be more susceptible to disturbances resulting in significant amplification of shear layer instabilities. The fact that the $32^{\circ} / 20^{\circ}$ $\left(\alpha_{11} / \alpha_{3}\right)$ case resulted in higher gap flow where $u_{\mathrm{g}}$ is $1.6 \mathrm{U}_{\infty}$ than the $26^{\circ} / 20^{\circ}$ case where $\mathrm{u}_{\mathrm{g}}$ is $1.55 \mathrm{U}_{\infty}$ (lower) implies that the high frequency noise is not solely due to these local flow changes. This result potentially indicates the influence of geometrical changes. It can be expected that different main element settings may result in different initial gap flow conditions.

Coherence data from Kulites in the slat cove show increasingly high coherence levels in the $25 \mathrm{kHz}$ region between pressure side sensors 146 and 145 as the slat angle is increased from $10^{\circ}$ to $30^{\circ}$. Figure 12 shows this high level of coherence for the $26^{\circ} / 30^{\circ}$ case. This relatively high level of coherence is not maintained between slat pressure-side sensors 146 (slat cove) and 144 (near the trailing edge) or between any of the sensors on the slat suction side. This suggests a more local acoustic event.

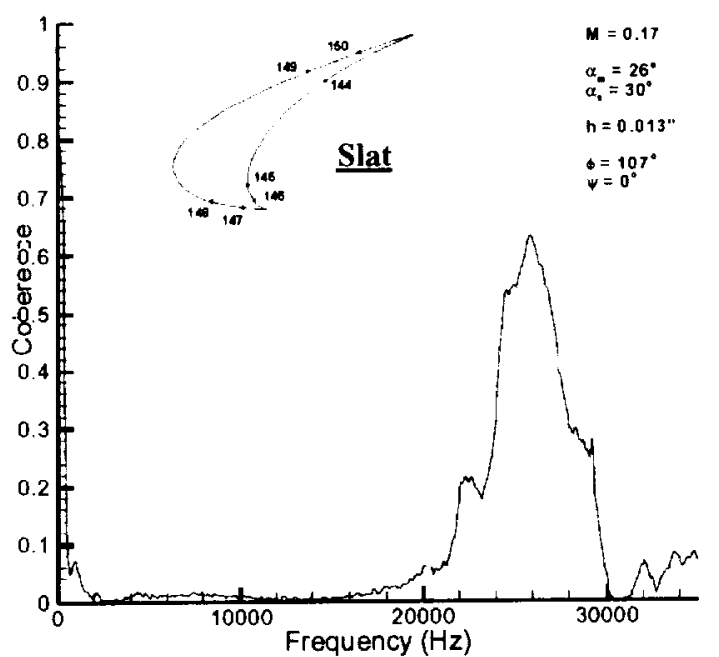

Figure 13 Coherence between cove sensors 146-145 for $\alpha_{\mathrm{m}}=26^{\circ} / \alpha_{\mathrm{s}}=30^{\circ}$.

\section{Effect of Gap Reduction}

The effect of reducing the gap size is shown in Figure 14. Reducing the gap size by $\sim 1 \%$ of the main element chord significantly reduced the noise in the lower frequencies and completely closing the gap resulted in noise level reductions at all frequencies reaching as high as $\sim 14 \mathrm{~dB}$. The 9-notch configuration does provide a significant amount of noise reduction below $10 \mathrm{kHz}$. Only when the gap is completely closed do significant noise reductions occur at the higher frequency. The gap closing potentially chokes the pressure-side flow to the point that the cove behaves as a plenum where key noise producing structures cannot form or are attenuated. Noise reduction is expected if this source is a result of edge scattering. 


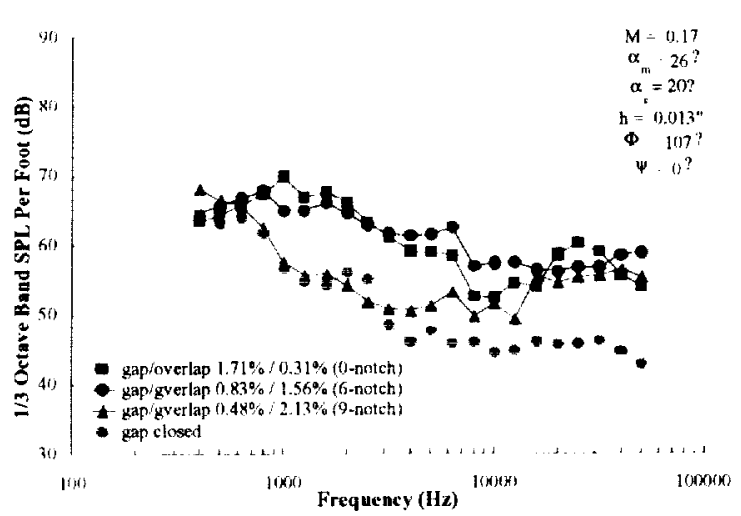

Figure 14 Baseline noise for varying gap/overlap.

\section{Flow Field Alterations}

Flow field modifications were made in an aftempt to discern the effect of Reynolds number and/or the state of the boundary layer on the measured sound. Figure 15 compares these results containing light tripping on the suction side, with the effect of heavier tripping ( 90 grit sand paper) on the pressure side, slat and main element, a tear drop cove insert, and the use of vortex generators on the pressure side. Figure 16 is an illustration of the grit and vortex generators. It appears that the additional tripping devices and the subsequent flow modifications did not significantly alter the spectral shape of the radiated sound field. Key aeroacoustic features from our model are captured. The more heavily tripped cases resulted in a slight increase in noise in the $1 \mathrm{kHz}$ to $10 \mathrm{kHz}$ range likely attributed to an increase in the shear layer instabilities associated with the cove vortex shedding. The teardrop insert provided some noise benefit in the low frequencies as well as the high frequency spectral hump region.

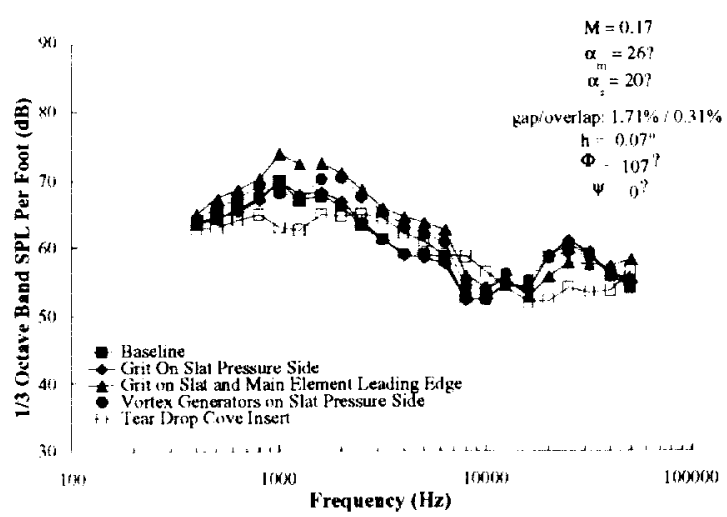

Figure 15 Effect of flow field modifications.

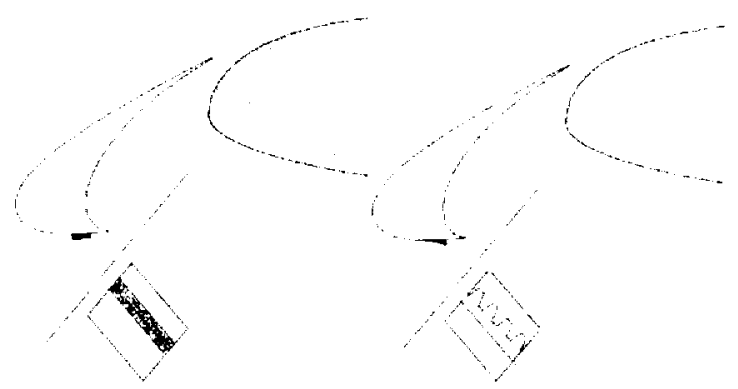

(a) Pressure side grit

(b) Pressure side VG's Figure 16 Boundary layer tripping devices.

\section{Mach Number Scaling}

Mach number variations for the $26^{\circ} / 20^{\circ}$ condition are shown in Figure 17. Characteristic of this data is the occurrence of a spectral peak followed by an approximate inverse square frequency law as consistently observed in the spectrum. Lilley ${ }^{20}$ has reported this spectrum roll-off as a common occurrence in may shear flows. In additional characteristic of the data is the presence of spectral hump at the higher frequencies as flow velocity increases. Computing Strouhal numbers based on stowed chord length and freestream flow velocity, it can be shown that the data from Figure 17 fall well within the Strouhal range reported by $\mathrm{Gou}^{7}$ (Figure 8). The Mach number scaling results to follow contain measured frequencies scaled by a Mach number ratio (data scaled to $M=0.17$ ).

The data in Figure 17 was normalized based on $\mathrm{M}^{5}$ and $\mathrm{M}^{4}$ power laws versus a frequency adjusted to flow Mach number in Figure 18 and Figure 19, respectively. This type of frequency scaling is essentially a Strouhal scaling where the characteristic length (e.g., slat chord) is assumed unchanged by the Mach number changes. The $\mathrm{M}^{5}$ scaling would result from simple scattering off the slat trailing edge. Figure 18 suggests $\mathrm{M}^{5}$ scaling provides a reasonably good collapse of the data at all frequencies. A better collapse of the data in the midfrequency range is found using a $\mathrm{M}^{4}$ power scaling, see Figure 19. Dobrzynski et. al. ${ }^{\text {is }}$ found that scaling their data with $\mathrm{V}^{4.5}$ provided the best collapse. Although $\mathrm{M}^{45}$ did not better fit the measured data here, it is clear that something lower than $\mathrm{M}^{5}$ may improve the collapse (at least in the mid-frequencies). A section titled Diagnostic using Prediction Simulation will illustrate that this Mach number scaling behavior is consistent with predictions of TE noise for isolated airfoils. 


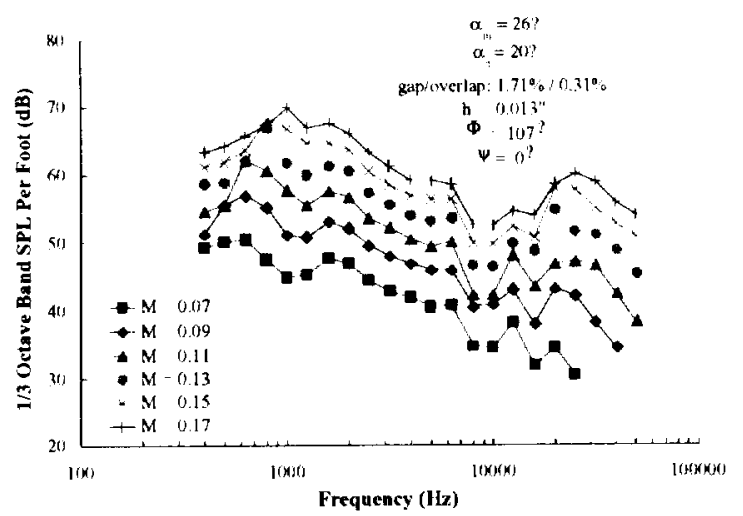

Figure 17 Mach number variations for $26^{\circ} / 20^{\circ}$.

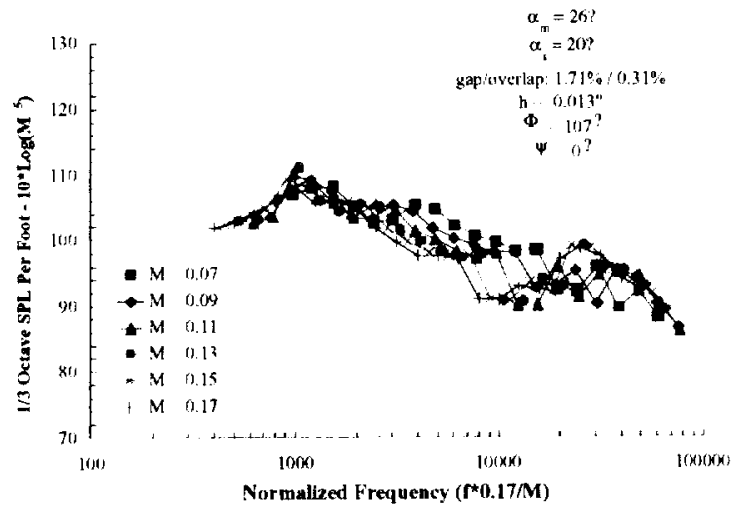

Figure $18 \mathrm{M}^{5}$ scaling of the baseline $26^{\circ} / 20^{\circ}$ data.

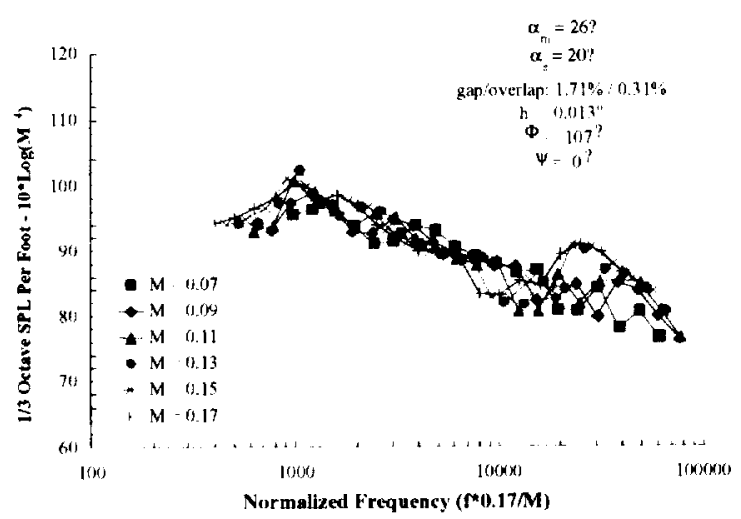

Figure $19 \mathrm{M}^{4}$ scaling of the baseline $26^{\circ} / 20^{\circ}$ data.

In a similar manner data for the $32^{\circ} / 20^{\circ}$ configuration are presented in Figure 20 and scaled with Mach number as $\mathrm{M}^{5}$ and $\mathrm{M}^{4}$ in Figure 21 and Figure 22, respectively. Similar trends found. The data appears to scale as something other than $\mathrm{M}^{5}$ and in fact for this particular configuration the mid and high frequencies better scale as $\mathrm{M}^{4}$.
Mach number scaling using a power lower than 4 did not improve the data collapse. As indicated, Guo's? OASPL data scaled between $\mathrm{M}^{2}$ and $\mathrm{M}^{3}$, which was attributed to the non-compact high frequency sources under consideration. Measured high frequency results scale better using $\mathrm{M}^{5}$ for the 0-notch TE configuration just presented and blunt edge configurations more clearly follow an $\mathrm{M}^{5}$ scaling. Both indicative of trailing edge noise.

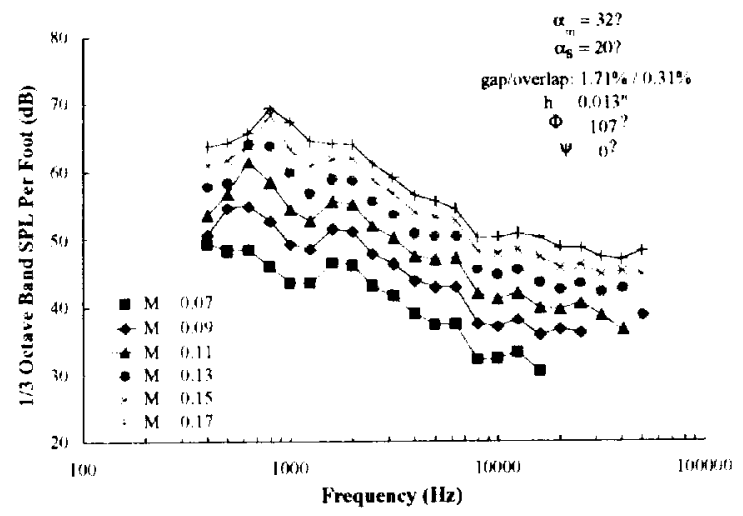

Figure 20 Mach number variations for $32^{\circ} / 20^{\circ}$ data.

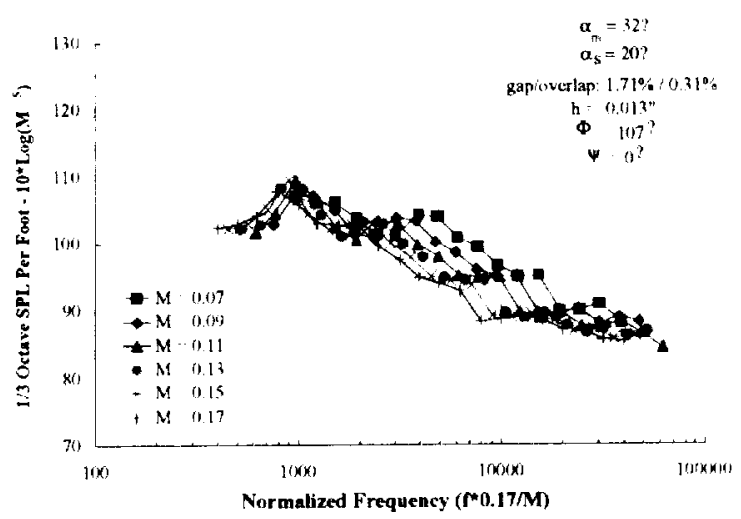

Figure $21 \mathrm{M}^{5}$ scaling of the $32^{\circ} / 20^{\circ}$ data.

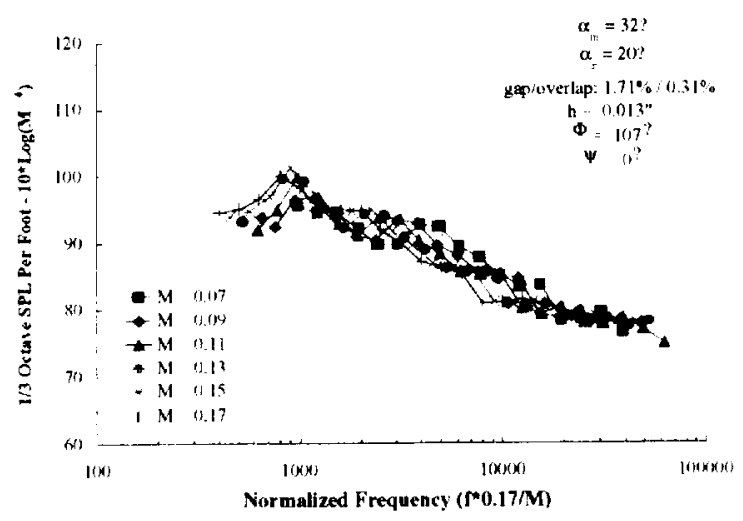

Figure $22 \mathrm{M}^{4}$ scaling of the $32^{\circ} / 20^{\circ}$ data. 


\section{Slat Trailing Edge Bluntness}

The $h=0.013$ " configuration was altered to investigate the effect of trailing edge bluntness on slat noise production by thickening and thinning the slat trailing edge, see Figure 23. Thinning the slat trailing edge was accomplished by extending a very thin, $0.005 "$, but sturdy strip of tape from the slat trailing edge.

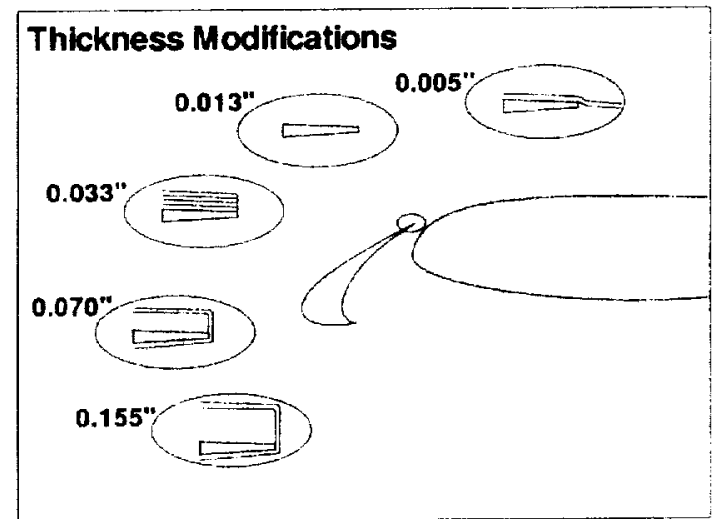

Figure 23 Slat trailing edge thickness modifications.

The $26^{\circ} / 20^{\circ}\left(\mathrm{h}=0.013^{\prime \prime}\right)$ slat acoustics varied with Mach number as shown in Figure 17. Figure 24 shows the effect of Mach number for a slat thickness of $h=$ 0.07 ". The thicker slat trailing edge introduced a relatively large peak in the spectrum that increases in frequency as the Mach number increases. The remaining spectral features remained unchanged for this condition indicating that predictions pertaining to this mechanism could be superimposed with the estimates of other prominent mechanisms. This is the subject of a future section.

Coherence data between sensors in the slat cove indicate values on the order of about $\gamma^{2} \sim 0.4-0.6$ in the vortex shedding region and comparable levels in the $22-25 \mathrm{kHz}$ range, similar to that indicated in Figure 13. Kulites near the trailing edge were covered or partially so. The sensors in the cove (specifically sensors 145,146 , and 147) clearly indicate the presence of a strong acoustic source emanating from the slat trailing edge. Interesting to note is that the phase difference between sensor 146 and 145 is essentially zero in the $12.5 \mathrm{kHz}$ range indicating that the acoustic wave front or pattern impinges upon these sensors nearly in phase.

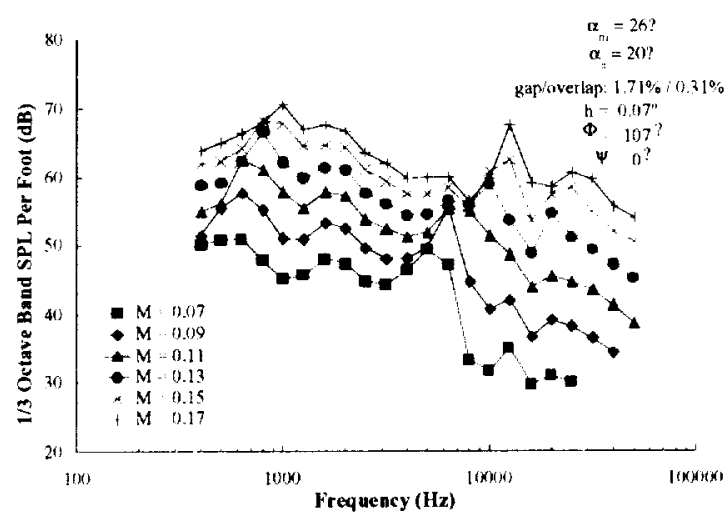

Figure 24 Effect of Mach number for $\mathrm{h}=0.07$ '.

Figure 24 is scaled as $\mathrm{M}^{5}$, see Figure 25 . As expected, the spectral peak associated with the added slat bluntness scales well with $\mathrm{M}^{\mathrm{s}}$. The scaling trends at frequencies other than the shedding frequency is consistent with that of Figure 18.

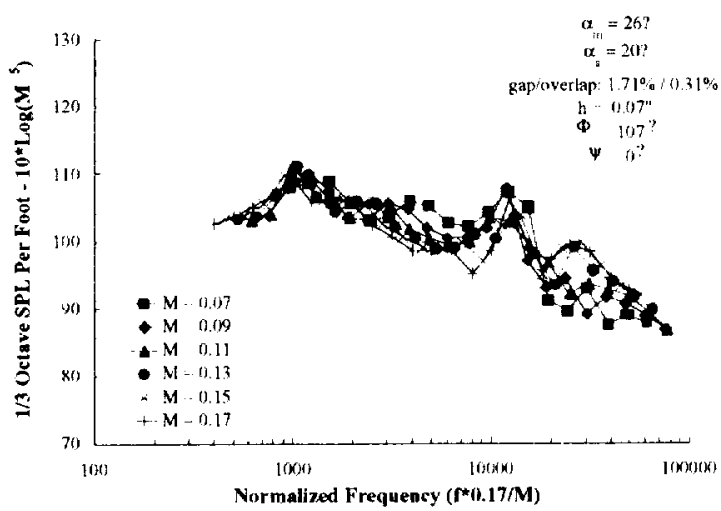

Figure $25 \mathrm{M}^{5}$ scaling of Figure 24 data.

The Mach number effects on a trailing edge thickness of $h=0.155^{\prime}$ are depicted in Figure 26 and the $M^{5}$ scaling of this data in Figure 27. As with the previous thickness condition, the bluntness adds a sharp peak to the baseline acoustic results in the mid frequency range that increasing with increasing Mach number. Peak frequencies occur at frequencies lower than the corresponding cases for $h=0.07$ " indicating a classical Strouhal scaling with trailing edge thickness (displacement thickness, momentum thickness, etc.). 


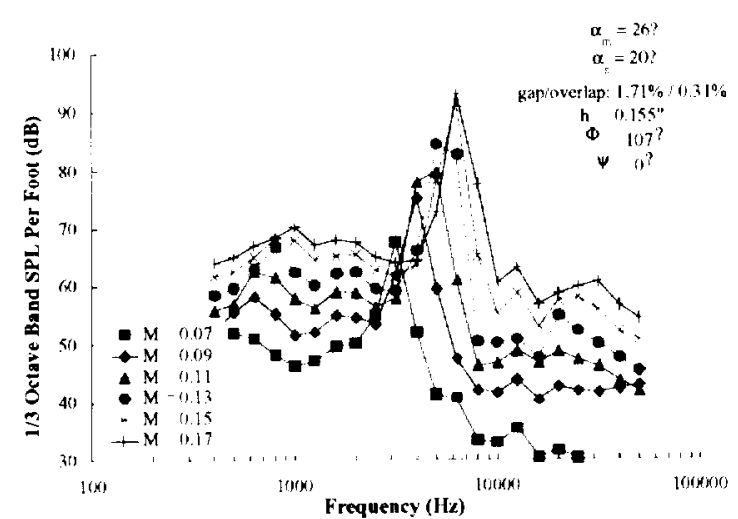

Figure 26 Effect of Mach number for $h=0.155$ ".

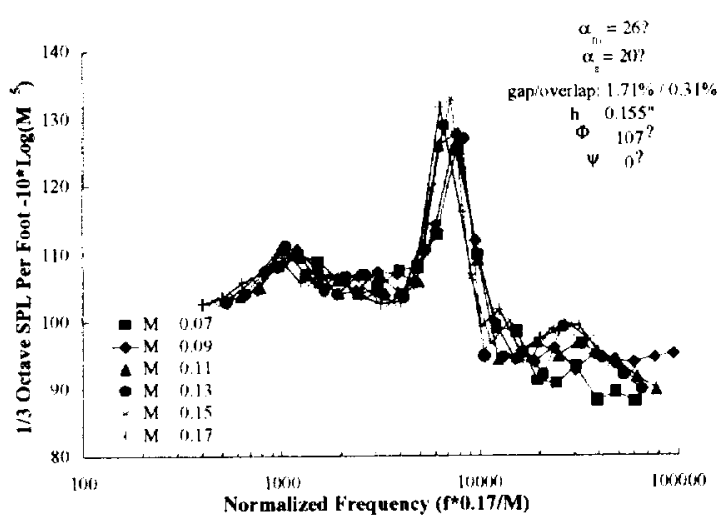

Figure $27 \mathrm{M}^{5}$ scaling of Figure 26 data.

The vortex-shedding peak resulting from the trailing edge bluntness is significantly elevated above the broadband levels of the spectrum compared to that of Figure 24 and Figure 25. This peak amplitude may be the result of acoustic reinforcement mechanism such as resonance or feedback at this condition. The wavelength of the reinforced signal requires a distance near 1 " for the $\mathrm{M}=0.17$ and $\mathrm{h}=0.155$ " condition that one could possibly tie to cove geometry. Similarly it could be argued that the other blunt configurations $(h<$ 0.155 ") could be suppressed at the shedding frequencies resulting in lower than expected amplitudes. Further investigation is needed to explain this behavior.

Coherence results for $\mathrm{M}=0.17$ and surface sensors 147 and 145 are presented in Figure 28 for $h=0.07$ " and $h$ $=0.155^{\prime \prime}$. This figure indicates very strong coherence $\gamma^{2} \sim 1$ for the bluntness peak and sharp coherence of $\sim$ 0.35 at $\sim 14 \mathrm{kHz}$ that is roughly twice the bluntness peak frequency for the thickest TE condition. The peak coherence level is reduced by $\sim 40 \%$ for the $h=0.07$ " TET. The phase from this sensor combination is indicated in Figure 29 it is found that both mechanisms propagate from the trailing edge region to the leading edge at an acoustic velocity (based on the separation distance between sensors). Coherence between sensor 144 (pressure side near the slat TE) and sensors in the cove region show essentially no coherence at the second peak frequency of Figure 28. Also interesting to note is that the coherence from sensors on the suction side of the slat indicates a relatively high level of coherence with sensors in the leading edge region. This is in contrast to the result of the $h=0.07$ " case where coherence levels where relatively weak between suction side and the leading edge sensors. This finding may implicate a resonance mechanism with harmonics associated with suction side instabilities that reinforce the $\mathrm{h}=0.155$ " condition.

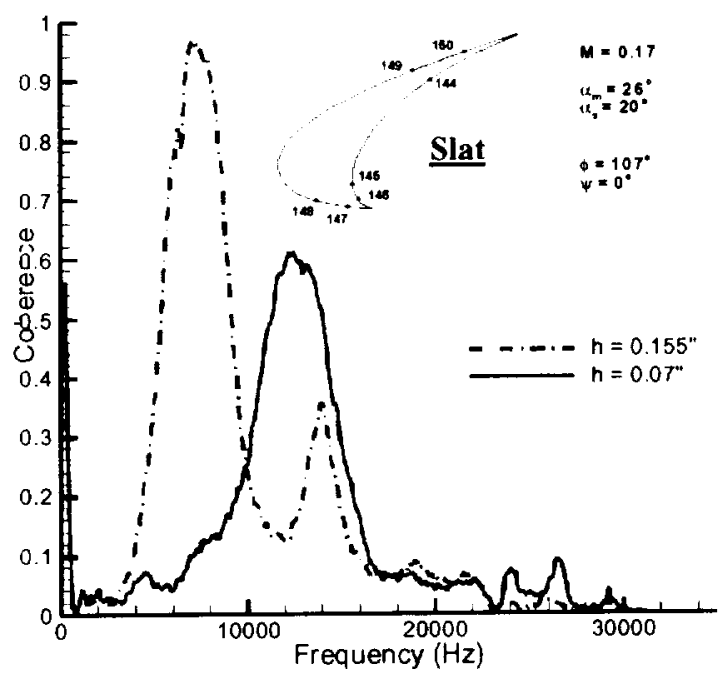

Figure 28 Coherence between cove sensors 147-145 in for $h=0.07^{\prime \prime}$ and $h=0.155^{\prime \prime}$.

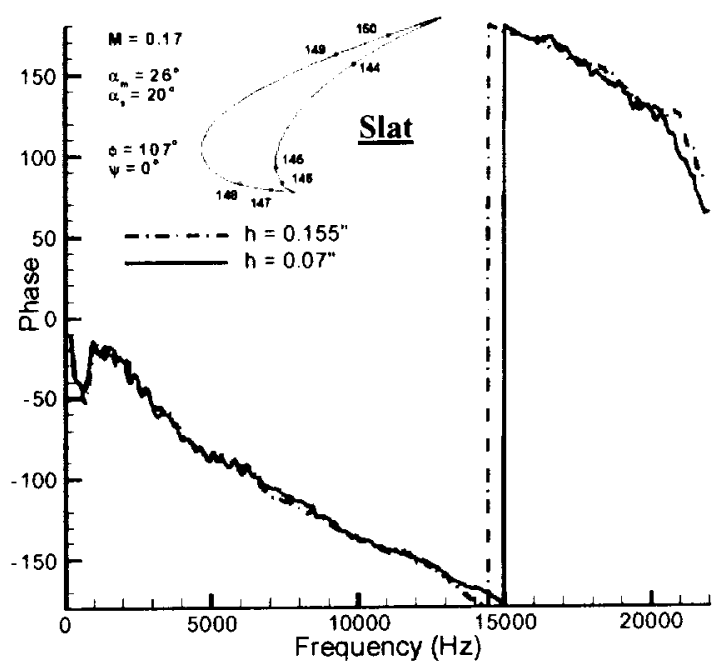

Figure 29 Phase relationship between sensors 147 and 145 for $h=0.07 "$ and $h=0.155^{\prime \prime}$. 
The effect of trailing edge thickness at a fixed Mach number, $M$, of 0.17 is shown in Figure 30 . Mid to low frequencies remain unchanged due to the added bluntness and the mid to high frequencies are affected only in the range where vortex shedding from the blunt trailing edge occurs. Strouhal scaling this data as $\mathrm{St}=$ $\mathrm{fh} / \mathrm{u}_{\mathrm{g}}$ results in a Strouhal number near $\mathrm{St} \sim 0.25$ at the shedding frequencies, slightly lower than $\mathrm{St}=0.3$ as reported by Lilley ${ }^{20}$. Based on this Strouhal number vortex shedding from the baseline thickness $h=0.013$ " and $\mathrm{h}=0.005$ " would occur well outside of our acoustic data range. The high frequency spectral hump present in the $25 \mathrm{kHz}$ range of our baseline data likely cannot be explained by blunt trailing edge noise.

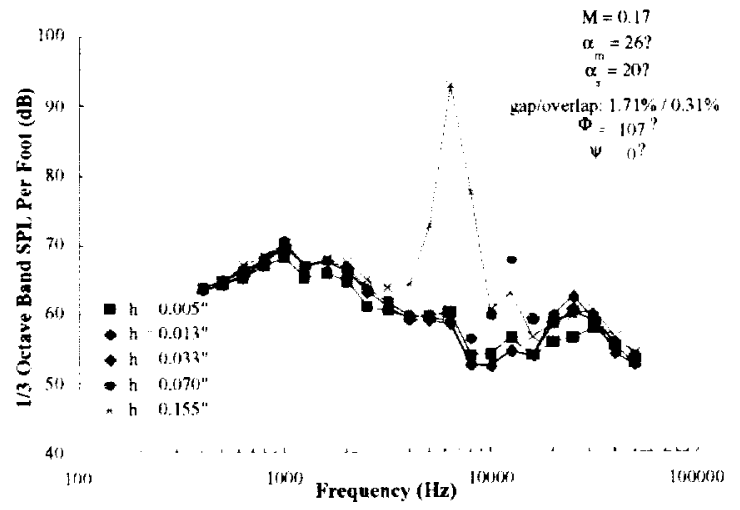

Figure 30 Effect of TET at $M=0.17$

\section{Directivity}

The acoustic measurements included positioning the SADA at multiple elevation angles relative to the nozzle centerline axis. Amplitude normalization is introduced that assumes a simple dipole utilizing adjusted angles from the shear layer refraction code and emission coordinates, see Brooks et. al. ${ }^{21}$.

Figure 31 shows the $26^{\circ} / 20^{\circ}$ directivity from pressure side elevation angles as indicated in Figure 6. The $56^{\circ}$ elevation angle data is excluded because noise maps indicate that the SADA at this position was not accurately pointed to the slat region. Noise levels continuously increase as the SADA position moves from the rear wing trailing edge position $\left(0^{\circ}\right.$ elevation in our nomenclature) toward the slat leading edge. The adjusted elevation range does not cover the entire pressure side arc, but it does appear that the peak overall levels may occur just beyond flyover.

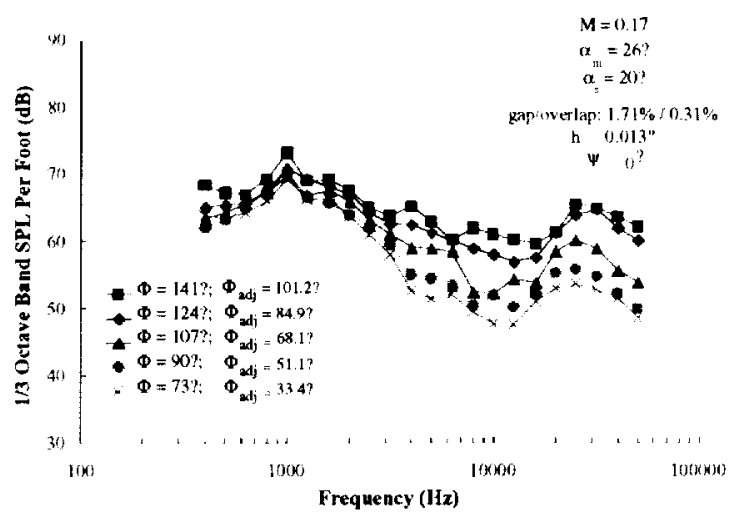

Figure 31 Elevation directivity of baseline.

Figure 31 shows the directivity normalization as applied to Figure 30 data. The simple dipole normalization does a good job of collapsing the higher frequencies. In Figure 30, the data at the low frequency end appear to collapse very well without normalization. As part of the SADA processing, all results are corrected to a 5 ' observer position from the source. The spectral characteristics in the low frequency may be interpreted as the result of a nearly omnidirectional sound source.

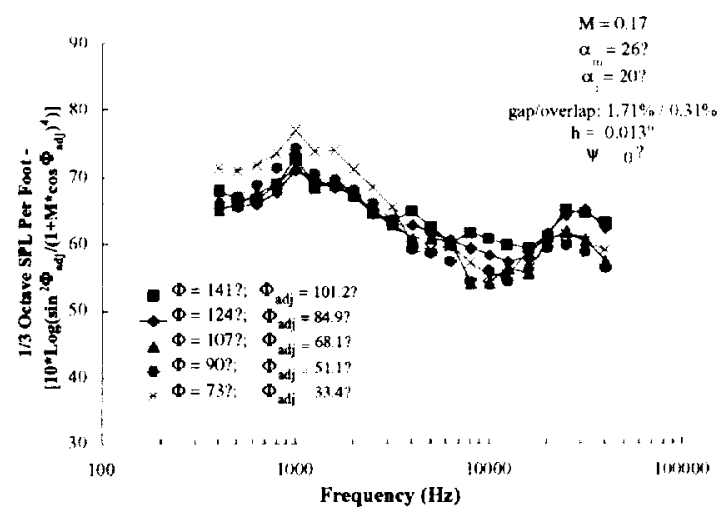

Figure 32 Directivity normalization of Figure 33 data.

Similar trends are observed for the 6-notch gap/overlap setting, see Figure 33 and Figure 34 for the uncorrected and corrected directivity results, respectively. As well as the thickest trailing edge configuration, i.e., $\mathrm{h}=0.155$ " (Figure 35 and Figure 36). The bluntness peak corrected levels illustrated in Figure 36 collapse to within a few $\mathrm{dB}$ with this correction. As previously indicated, only a few directivity plots are shown here. All the processed slat noise data to date has collapsed as indicated. The simple dipole normalization collapses the mid to high frequencies consistent with the expected radiation of the assumed edge scattering mechanisms. 


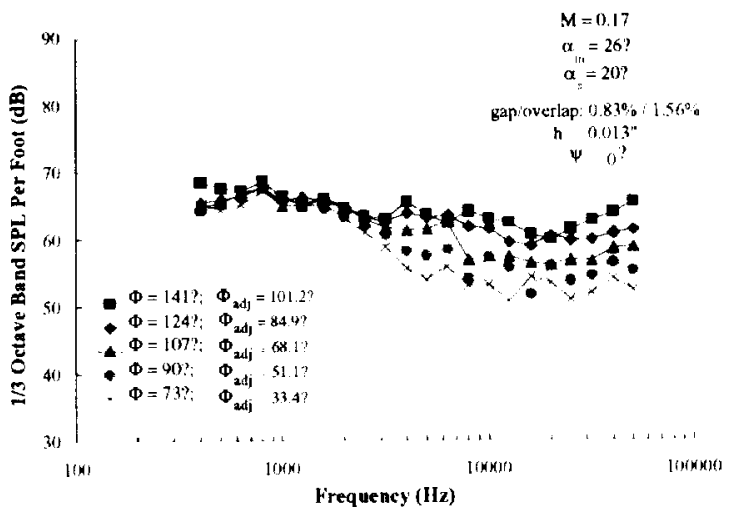

Figure 33 Elevation directivity for 6-notch setting.

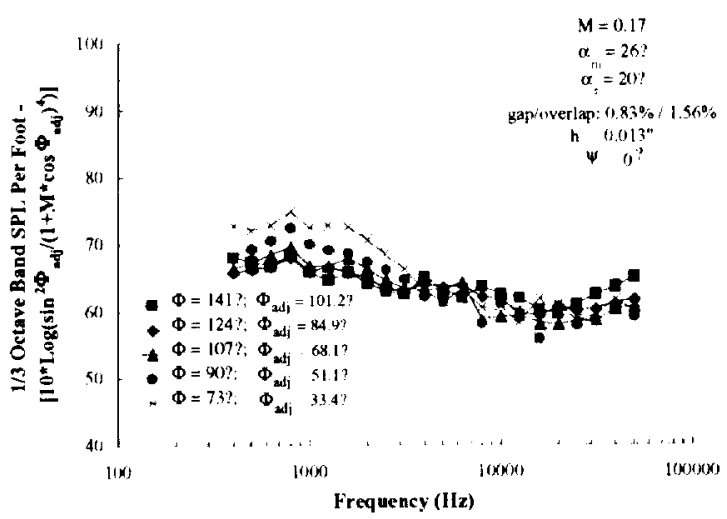

Figure 34 Directivity correction of Figure 36 data.

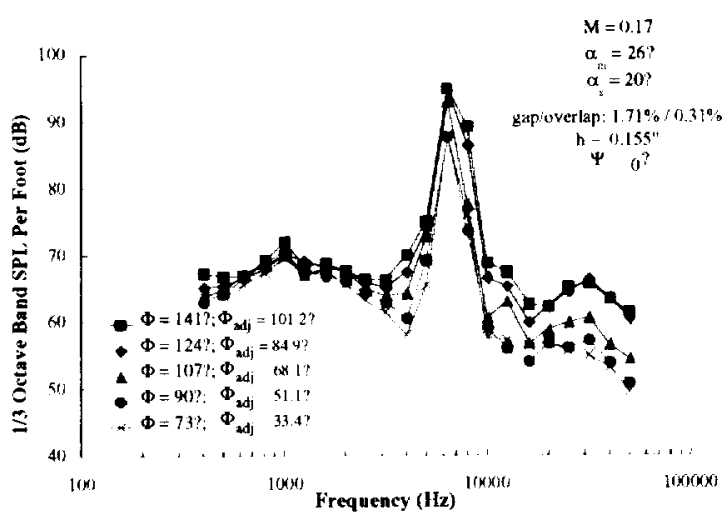

Figure 35 Elevation directivity for blunt TE.

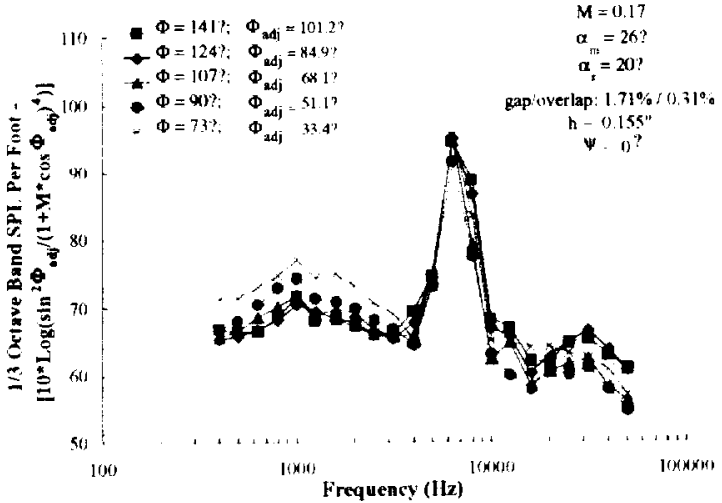

Figure 36 Directivity correction of Figure 38 data.

\section{Diagnostic using Prediction Simulation}

Although a prediction method for slat noise is not given here, an existing trailing edge noise prediction method can serve as a diagnostic to examine source components and their ability to explain the measured spectra. In this section, boundary layer thickness values and velocities of Table 3 are used in the airfoil self noise prediction codes of Brooks et $\mathrm{al}^{21}$ and comparisons are made to measured slat noise spectra.

The airfoil self noise codes contain models for turbulent boundary layer - trailing edge (TBL-TE) noise, laminar boundary layer - trailing edge (LBL-TE) noise, blunt trailing edge (BTE) noise, and tip noise. Figure 37 shows a "prediction" where the models used are that of the TBL-TE noise and BTE noise components. The "predictions" are for an observer that is 5 feet away and normal to the chord of an isolated airfoil (no accounting is made of slat geometry nor that the line of sight of the slat TE is blocked by the main element). A comparison can be made to the spectrum of the $\alpha_{\mathrm{m}}=26^{\circ} / \alpha_{\mathrm{s}}=20^{\circ}$ case with bluntness of thickness $\mathrm{h}=0.155^{\prime}$ for $\mathrm{M}=0.17$, shown in Figure 26 and Figure 30. In Figure 37 :

- The pressure side and suction side TBL-TE noise spectral components are predicted using $\delta_{p}^{*}, \delta_{s}^{*}$ (rather than using the code's internally generated values) and $u_{p}, u_{s}$ values from Table 3 .

- A lower frequency spectral component to TE noise is included, which is suggested here to be representative of turbulence within the cove region passing the TE. This is calculated in the manner of pressure side TBLTE noise using the average velocity across the gap, $u_{\mathrm{g}}$, from Table 3 and a selected value of $\delta_{p}{ }^{*}=0.25$ ". (Note that this $\delta_{p}^{*}$ value in the code is indicative of a turbulent scale of 1 " to 2.5 ", because of the normal $\delta^{*} / \delta$ relationship)

- For the spectral component due to bluntness, the BTE model uses $h, \delta_{p}^{*}$ and $\delta_{s}^{*}$, a $\Psi$ factor in the code 
equaling zero for a cusped TE, and a stream velocity $u_{a v}$ from Table 3.

- The total spectrum shown in Figure 37 is the sum of the separate components.

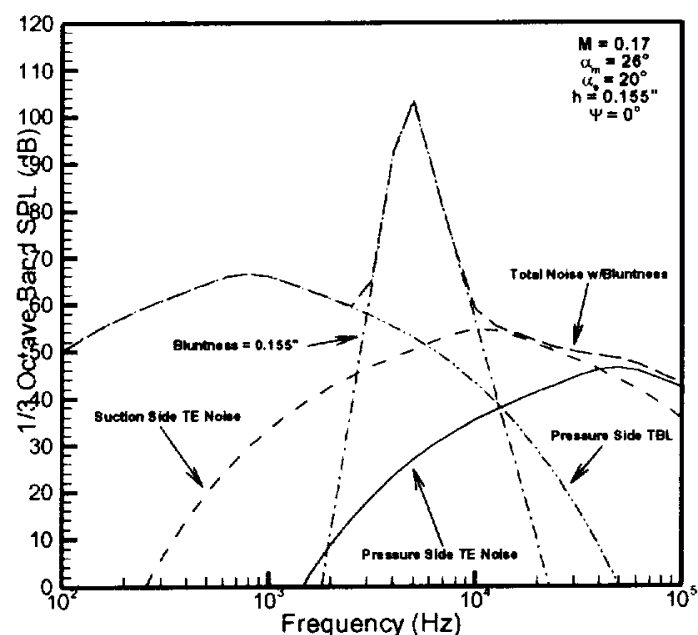

Figure 37 Self noise prediction of blunt TE.

Additional calculated results are shown in Figure 38 and Figure 39 of spectra for the baseline cases where the BTE term is excluded (this is subsequently discussed). The same calculation procedure involving Table 3 was used. These spectra can be compared to those of Figure 11 and Figure 12, respectively. Also, in Figure 40 , the $\alpha_{m}=26^{\circ} / \alpha_{s}=20^{\circ}$ baseline case for different tunnel speeds are calculated and scaled in the manner of Figure 18 for the corresponding measured spectra.

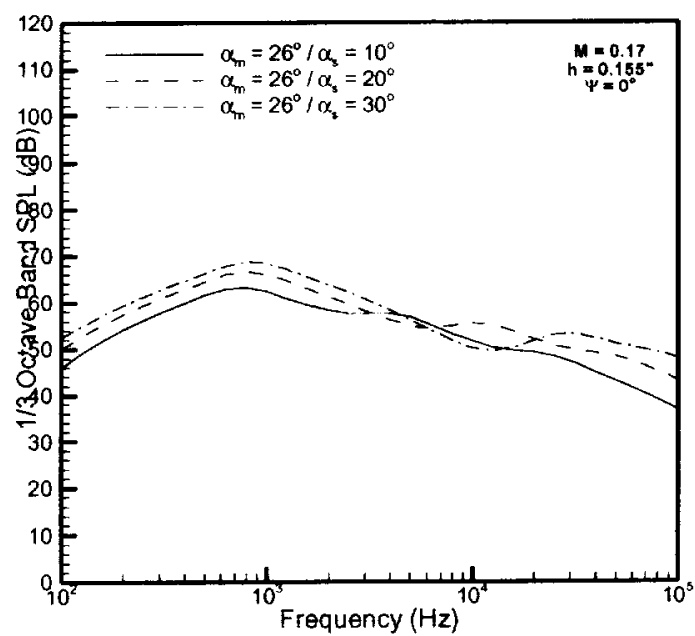

Figure 38 Self noise predictions of the effect slat angle variation for $\alpha_{m}=26^{\circ}$.

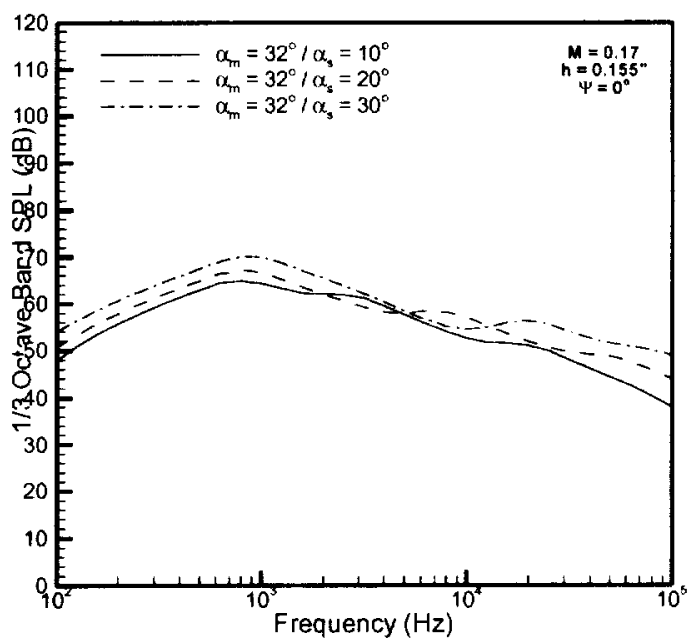

Figure 39 Self noise predictions of the effect slat angle variation for $\alpha_{111}=32^{\circ}$.

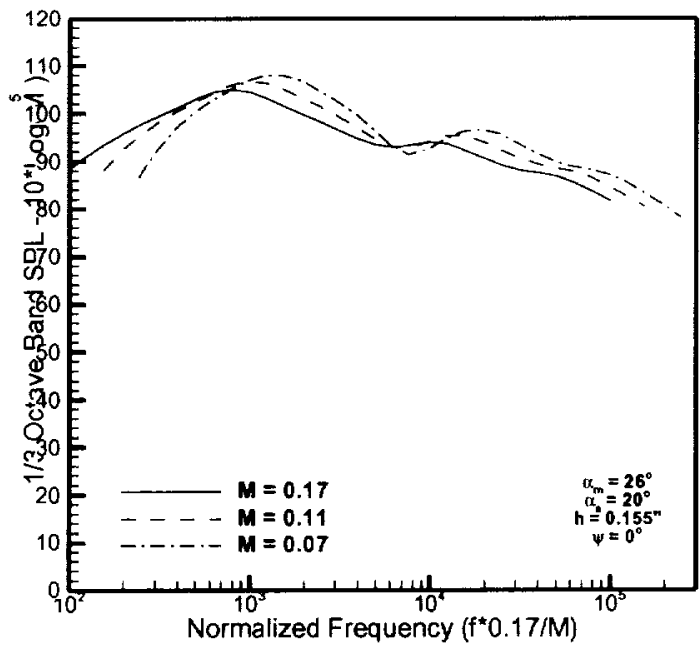

Figure 40 Self noise predictions of the effect of Mach number using $\mathrm{M}^{5}$ noise scaling.

Clearly the calculations are greatly simplified with regard to flow detail and turbulence structure definitions, as well as geometry related acoustic effects. Still, comparing these "predictions" with data measured indicate some key mechanisms are likely captured.

- First, the low frequency component, thought by the authors to be logically related to TE noise due to the passage of cove region turbulence, appears well represented in many respects using a straight-forward application of the self noise code. But in comparing Figure 38 and Figure 39 to Figure 11 and Figure 12, respectively, this low frequency component is seen to be influenced by $\alpha_{m}$ and $\alpha_{s}$ changes, apparently because 
of turbulent structure changes not being accounted for in the code application.

- Second, the calculated TBL related TE noise appears to account for at least a portion of the higher frequency noise. In this high frequency range however, there seems to be a strong additive noise or noise reinforcing effect from the cove region depending on the $\alpha_{\mathrm{in}} / \alpha_{\mathrm{s}}$ condition. In a compatible result with the measurements (comparing Figure 40 to Figure 18) the velocity scaling of the "predicted" spectra show the same observed behavior as seen for the measurement spectra, that the scaling does not closely follow the $5^{\text {th }}$ power scaling law.

- Third, by comparing Figure 37 and Figure 30, one sees a general agreement with regard to the bluntness noise (BTE) calculation. For other " $h$ " thicknesses, BTE calculations have shown generally good (if not somewhat low) peak-frequency predictions. However, the levels were substantially higher than measured. As seen in Figure 30, the measured levels at the smaller thicknesses are seen to be subdued compared to what one may expect, given the high peak level for $\mathrm{h}=0.155 \%$, A number of factors could affect this phenomenon. The TE flow behavior was not fully measured and flow skew and other distortions could serve to suppress the vortex shedding. Also, aeroacoustic feedback could suppress or reinforce the vortex shedding dependent on the frequencies. Such effects need study.

\section{Summary and Conclusions}

An extensive experimental investigation has been conducted in the QFF of NASA LaRC pertaining to slat noise. Unique array processing was developed and utilized to focus acoustic results on the distributed nature of a 2-D wing and slat model. The measured noise levels have been presented with respect to 1 -foot span of the slat. The focus of this investigation was primarily on the broadband nature of slat noise and tying the measured noise to sources to key slat flow features. Slat trailing edge bluntness effects were also considered. A number of germane aeroacoustic scaling results and slat acoustics have been identified. In addition, a diagnostic tool for slat noise provided successful examination of key slat noise source. Key results are summarized.

- An increase in slat angle increases the high frequency noise levels where a broad spectral hump was observed. High coherence levels were found in this frequency range between cove sensors. This noise increase coincides with an increase in velocity through the slat gap. Predictions using measured BL parameters indicate that the high frequency spectral hump can be partially attributed to TBL-TE noise most likely from the slat pressure side.

- Completely closing off the slat gap resulted in noise level reductions as high as $14 \mathrm{~dB}$. The cove teardrop insert was also shown to be an effective noise reduction device at most frequencies.

- Mach number scaling using $5^{\text {th }}$ power provided the best collapse of the noise data providing strong evidence of self-noise (edge scattering) mechanisms.

- Thickening the slat trailing edge resulted in a highly coherent vortex shedding source. Strouhal scaling of the bluntness data with $\mathrm{h}$ and $\mathrm{u}_{\mathrm{g}}$ results in $\mathrm{St}$ of $\sim 0.25$ at the vortex shedding frequency.

- The thickest bluntness condition was well predicted using the airfoil self noise code of Brooks et al. ${ }^{21}$. Other slat trailing edge thicknesses produced slightly lower than expected bluntness noise that may implicate damping from an aeroacoustic feedback mechanism at the vortex shedding frequencies.

- A simple dipole with convection effects was used to normalize the elevation angle directivity data. This normalization provided a good collapse of the data at most frequencies. The very low frequencies collapsed better without normalization potentially implying the presence of an omnidirectional source in addition to the self-noise sources already identified.

- The diagnostic tool appears to capture most of the salient features and trends of the measured noise data.

\section{Acknowledgements}

The authors wish to gratefully acknowledge Dr. Florence V. Hutcheson from the Aeroacoustics Branch at NASA LaRC for her assistance in some of the analysis. The authors also gratefully acknowledge Daniel J. Stead of Lockheed-Martin for data acquisition, data processing, software development, and numerous other miscellaneous tasks and support.

\section{References}

1. Macaraeg, M.G., "Fundamental Investigations of Airframe Noise", AIAA Paper 98-2224, June 1998.

2. Davy, R., and Remy, H., "Airframe Noise Characteristics on a $1 / 11$ Scale Airbus Model", AIAA Paper 98-2335, June 1998.

3. Hayes, J.A., Home, C.W., Soderman, P.T., and Bent, P.H., "Airframe Noise Characteristics of a 4.7\% Scale DC-10 Model ", AIAA Paper 97-1594$C P, 3^{\text {rd }}$ AIAA/CEAS Aeroacoustics Conference and Exhibit, Atlanta, GA, May, 1997.

4. Dobrzynski, W., Nagakura, K., Gehlhar, B., and Buschbaum, A., "Airframe Noise Studies on Wings

17 
with Deployed High-Lift Devices ", AlAA Paper 99-1805, $5^{\text {th }}$ AIAA/CEAS Aeroacoustics Conference and Exhibit, Bellevue, WA, May, 1999.

5. Guo, Y.P., Hardy, B., Bent, P., Yamamoto, K., and Joshi, M., "Surface Pressure Fluctuations on DC10 High Lift System and their Correlation with Far Field Noise", NASl-20103, Task 2, NASA CRAD9310-4872, 1998.

6. Guo, Y.P., Hardy, B., Bent, P., Yamamoto, K., and Joshi, M., "Noise Characteristics of DC-10 Aircraft High Lift Systems", NASI-20103, Task 2, NASA CRAD-9310-4893, 1998.

7. Guo, Y.P., "A Discrete Vortex Model for Slat Noise Generation", AIAA Paper 2001-2157, $7^{\text {th }}$ AIAA/CEAS Aeroacoustics Conference, Maastricht, Netherlands, May, 2001.

8. Thomas, F.O., Nelson, R.C., and Lui, X., "Experimental Investigation of the Confluent Boundary Layer of a High-Lift System", AIAA Journal, Volume 38, Number 6, pp. 978-988, June, 2000.

9. Moriarity, P.J., and Heineck, J.T., "PIV Measurements Near a Leading Edge Slat" Proceedings of the $3^{\text {rd }}$ International Workshop on PIV 1999.

10. Storms, B.L., Hayes, J.A., Moriarity, P.J., and James, R.C., "Aeroacoustic Measurements of Slat Noise on a Three-Dimensional High-Lift System", AIAA Paper 99-1957, $5^{\text {th }}$ AIAA/CEAS Aeroacoustics Conference and Exhibit, Bellevue, WA, May, 1999..

11. Guo, Y.P., "A Model for Slat Noise Generation", AlAA Paper 97-1647, $3^{\text {rd }}$ AIAA/CEAS Aeroucoustics Conference and Exhibit, Atlanta, GA, May, 1997.

12. Khorrami, M.R., Berkman, M.E., Choudhari, M., Singer, B.A.,Lockard, D.P., and Brentner, K.S., "Unsteady Flow Computations of a Slat with a Blunt Trailing Edge ", AIAA Paper 99-1805, $5^{\text {th }}$ AIAA/CEAS Aeroacoustics Conference and Exhibit, Bellevue, WA, May, 1999.

13. Singer, B.A., Lockard, D.P., Brentner, K.S.Khorrami, M.R., Berkman, M.E., and Choudhari, M, "Computational Aeroacoustic Analysis of Slat Trailing Edge Flow", AIAA Paper 99-1802, $5^{\text {th }}$ AIAA/CEAS Aeroacoustics Conference and Exhibit, Bellevue, WA, May, 1999.
14. Khorrami, M.R., Singer, B.A., Berkman, M.E., "Time Accurate Simulations and Acoustic Analysis of Slat Free-Shear Layer", ALAA Paper 01-2155, $7^{\text {th }}$ ALAA/CEAS Aeroacoustics Conference and Exhibit, Maastricht, Netherlands, May, 2001.

15. Dobrzynski, W. and Pott-Pollenske, M., "Slat Noise Source Studies for Farfield Noise Prediction ", AIAA Paper 2001-2158, $7^{\text {th }}$ AIAA/CEAS Aeroacoustics Conference and Exhibit, Maastricht, The Netherlands, May, 2001.

16. Humphreys, W.M., Brooks, T.F., Hunter, W.W., and Meadows, K.R., "Design and Use of Microphone Directional Arrays for Aeroacoustic Measurements", ALAA Paper 98-0471, 36st Aerospace Science Meeting and Exhibit, Reno, NV, January, 1998.

17. Meadows, K.R., Brooks, T.F., Humphreys, W.M., Hunter, W.W., and Gerhold, C.H., "Aeroacoustic Measurements of a Wing-Flap Configuration", AlAA Paper 97-1595, $3^{\text {rd }}$ AIAA/CEAS Aeroacoustics Conference, Atlanta, GA, May, 1997.

18. Brooks, T.F., and Humphreys, W.M., "Effect of Directional Array Size on the Measurement of Airframe Noise Components", AIAA Paper 99$1958,5^{\text {th }}$ Aeroacoustics Conference, Bellevue, WA, May, 1999.

19. Brooks, T.F., and Humphreys, W.M., "Flap Edge Aeroacoustic Measurements and Predictions", ALAA paper 2000-1975, 6th AIAA/CEAS Aeroacoustics Conference, Lahaina, Hawaii, June, 2000.

20. Lilley, G.M., "The Prediction of Airframe Noise and Comparison with Experiment", Journal of Sound and Vibration, 239(4), pp. 849-859, 2001.

21. Brooks, T.F., Pope, D.S., Marcolini, M.A., "Airfoil Self-Noise and Prediction", NASA Reference Publication 1218, July, 1989. 